\title{
Using RIXS to Uncover Elementary Charge and Spin Excitations
}

\author{
Chunjing Jia, ${ }^{1, *}$ Krzysztof Wohlfeld, ${ }^{1,2 \dagger}$ Yao Wang, ${ }^{1,3}$ Brian Moritz, ${ }^{1}$ and Thomas P. Devereaux ${ }^{1,4}$ \\ ${ }^{1}$ Stanford Institute for Materials and Energy Sciences, SLAC National Laboratory and Stanford University, \\ Menlo Park, California 94025, USA \\ ${ }^{2}$ Institute of Theoretical Physics, Faculty of Physics, University of Warsaw, \\ Pasteura 5, PL-02093 Warsaw, Poland \\ ${ }^{3}$ Department of Applied Physics, Stanford University, Stanford, California 94305, USA \\ ${ }^{4}$ Geballe Laboratory for Advanced Materials, Stanford University, Stanford, California 94305, USA
}

(Received 17 October 2015; revised manuscript received 6 April 2016; published 13 May 2016)

Despite significant progress in resonant inelastic x-ray scattering (RIXS) experiments on cuprates at the $\mathrm{Cu} L$-edge, a theoretical understanding of the cross section remains incomplete in terms of elementary excitations and the connection to both charge and spin structure factors. Here, we use state-of-the-art, unbiased numerical calculations to study the low-energy excitations probed by RIXS in the Hubbard model, relevant to the cuprates. The results highlight the importance of scattering geometry, in particular, both the incident and scattered $\mathrm{x}$-ray photon polarization, and they demonstrate that on a qualitative level the RIXS spectral shape in the cross-polarized channel approximates that of the spin dynamical structure factor. However, in the parallel-polarized channel, the complexity of the RIXS process beyond a simple two-particle response complicates the analysis and demonstrates that approximations and expansions that attempt to relate RIXS to less complex correlation functions cannot reproduce the full diversity of RIXS spectral features.

DOI: 10.1103/PhysRevX.6.021020

Subject Areas: Computational Physics,
Condensed Matter Physics,
Strongly Correlated Materials

quasiparticles can move through the solid. Unfortunately, there are not that many techniques that can perform this task. Perhaps the most well known and most widely used is inelastic neutron scattering (INS). However, this technique has a number of limitations (requirement of large sample sizes, limited dynamic range, availability of research reactors, or spallation sources). Other techniques such as optical Raman scattering and nonresonant inelastic x-ray scattering (IXS) also can provide some information about multimagnon, phonon, and electronic excitations. Particular attention has been devoted recently to the development of resonant inelastic x-ray scattering (RIXS), a complementary technique for understanding the energy and dispersion of elementary excitations, including electronic, lattice, magnetic, and dipole forbidden orbital excitations.

RIXS is an experimental technique in which the transferred energy, momentum, and polarization associated with incident and scattered x-ray photons can be measured and analyzed to reveal information about the elementary excitations of a system [1,2]. RIXS possesses atomic specificity with incoming photons resonantly tuned to a specific atomic absorption edge. For transition metal $L$ and $K$ edges in the soft and hard $\mathrm{x}$-ray regimes, these $\mathrm{x}$-ray photons carry momentum comparable to electronic crystal momenta, making RIXS a particularly unique and powerful tool for characterizing excitations across a significant portion of the electronic Brillouin zone. Combined with 
additional advantages such as bulk sensitivity and polarization control, RIXS has emerged as an influential technique for studying various condensed matter materials.

Although RIXS by nature has the ability to measure elementary excitations for numerous materials, it has shown an especially strong ability for characterizing elementary excitations in transition-metal oxides which manifest strong correlations [3-21]. In particular, RIXS has been shown to be quite useful for the study of high-temperature superconducting materials: As magnetic fluctuations were proposed as candidates for the superconducting pairing glue, obtaining their dispersion relation can be crucial for unraveling the mystery of high $T_{c}$ [22].

The direct RIXS process consists of two dipole transitions, as shown schematically in Fig. 1 for the $\mathrm{Cu} L$ edge. In the first step, an incoming photon excites the ground state by promoting an electron from a filled core shell $(\mathrm{Cu} 2 p)$ into the valence shell $(\mathrm{Cu} \mathrm{3d})$. An intermediate state manifold forms following the charge and spin shakeup that accompany the introduction of a local core-hole potential and this new carrier in the valence shell. In the second step, an electron from the valence shell, possessing appropriate atomic character, fills the core hole, accompanied by an outgoing photon, which leaves the system in an excited final state.

Because of the complexity of this process and the presence of the intermediate state, an interpretation of RIXS spectra has been hindered by an incomplete understanding of how it may be related to other, more fundamental, response functions governing spin, charge, lattice, and orbital excitations. In addition, it is well known that polarization plays a key role in understanding selective excitations in Raman spectroscopy [23]; however, a full polarization analysis involving both incident and scattered $\mathrm{x}$ rays has become possible now in experiment, and it is only shown to be important theoretically for spin-flip excitations [24]. One would expect that a more complete understanding, at a fundamental level, could be obtained by analyzing the full theoretical RIXS cross section, accounting for the influence of both the incident and scattered light polarization for a given experimental scattering geometry.

To better understand the RIXS cross section from a theoretical perspective, here we explore a rather general question: What low-energy excitations are measured by RIXS, particularly in cuprates at the $\mathrm{Cu} L$ edge? There certainly are a number of ways to address this question analytically and numerically. Here, our interest is confined to the low-energy excitations, which involve spin and/or charge degrees of freedom, neglecting both lattice and higherenergy orbital or charge-transfer excitations in this work. Our aim is to qualitatively understand which excitations are encoded in the RIXS cross section, as well as any connections to both spin and charge-dynamical structure factors, under different scattering conditions; we quantitatively compare numerical estimates of the full RIXS cross section with various approximations to tease out this information.

Naively, one may expect the $\mathrm{Cu} L$-edge RIXS cross section to be the resonant analog of the hard x-ray nonresonant IXS [25,26], whose response is the chargedynamical structure factor. It then may seem counterintuitive that RIXS at the $\mathrm{Cu} L$ edge rose to prominence for the successful empirical measurement of the spin (magnon) dispersion in undoped cuprates, making it a complementary experimental probe to the well-established inelastic neutron scattering [5,6]. With theoretical support [24,27], experiments on doped cuprates proved sensitive to magnetic excitations, with a similar cross section to the spin dynamical structure factor, regardless of doping level [8-18]. These observations were interpreted in terms of persistent magnetic excitations up to an unexpectedly high doping level. However, this semiempirical connection to the spin dynamical structure factor has been based primarily on approximate theoretical and numerical treatments for the full RIXS cross section. The fast collision approximation [28-30] and the effective operator approach [31] suggested that only single magnon excitations or $S(\mathbf{q}, \omega)$ should be measured by RIXS at the $\mathrm{Cu} L$ edge $[27,31,32]$. More sophisticated treatments-the ultrashort core-hole lifetime (UCL) expansion [33-35] and the UCL-inspired ansatz [36] - highlighted that RIXS should also be sensitive to bimagnon excitations. A further extension of UCL also pointed out the importance of three-magnon excitations [37]. However, these studies made no explicit comparison between the approximations and the full RIXS cross section, nor have they concentrated on the sensitivity of RIXS to the charge dynamical structure factor.

Perhaps more importantly, these approximations suffer from several severe limitations. First, the fast collision approximation (or the effective operator approach) $[27,31,32]$ relies on an estimation of the dynamics only at the site where the core hole has been created in the intermediate state. However, the intermediate state is drawn from a manifold of states, which differ from the ground state not only locally, at the site where a core hole is created, but also on neighboring sites due to hopping or spin exchange associated with the dynamical screening process. As a result, and especially upon doping, this approximation fails to capture key elements of the full RIXS process. Second, the UCL approximation relies on the assumption that the energies of the intermediate-state manifold are much smaller than the inverse core-hole lifetime $\Gamma$, which allows an approximation based on only the first few (two) terms in the UCL Taylor-series-like expansion. However, this assumption need not hold, especially in "itinerant," doped systems where a number of intermediate states may have energies $\propto t \sim \Gamma$, making the UCL a nonconvergent approximation.

In this paper, we study the low-energy excitations of RIXS at the $\mathrm{Cu} L$ edge in an unambiguous way by 
numerically evaluating the cross section for a twodimensional Hubbard model comprising "effective" $\mathrm{Cu}$ $3 d$ orbitals supplemented by local $\mathrm{Cu} 2 p$ core levels. Using exact diagonalization, which previously was applied to the study of paramagnons in cuprates [24], we compare the exact RIXS cross sections to approximate cross sections for the same model using the same method. The intrinsically correlated nature of our Hamiltonian distinguishes this study from the analytically exact RIXS calculations for the case of completely uncorrelated electrons [38]. In the next section, we present and compare numerical results for the exact and approximate RIXS cross sections and discuss content of the excitations and consequences for experiments. The paper ends with conclusions and appendixes that contain details and longer derivations.

\section{NUMERICAL RESULTS}

\section{A. Exact RIXS cross section}

The RIXS cross section at the $\mathrm{Cu} L$ edge is $[1,32]$

$$
I_{\mathbf{e}}(\mathbf{q}, \omega)=\sum_{f}\left|\left\langle f\left|O_{\mathbf{q}, \mathbf{e}}\right| i\right\rangle\right|^{2} \delta\left(\omega+E_{i}-E_{f}\right),
$$

where $|i\rangle(|f\rangle)$ is the initial (final) state of the system in the RIXS process with energy $E_{i}\left(E_{f}\right)$, transferred momentum (energy loss) is $\mathbf{q} \equiv \mathbf{k}_{i}-\mathbf{k}_{f}\left(\omega \equiv \omega_{i}-\omega_{f}\right)$, where $\mathbf{q}_{i}$ and $\mathbf{q}_{f}\left(\omega_{i}\right.$ and $\left.\omega_{f}\right)$ are the incoming and outgoing photon momenta (energy), and $\mathbf{e}=\mathbf{e}^{i} \cdot\left(\mathbf{e}^{f}\right)^{\dagger}$ is the tensor that describes the incoming $(i)$ and outgoing $(f)$ photon polarizations. Here, the operator $O_{\mathbf{q}, \mathbf{e}}=1 / \sqrt{N} \sum_{\mathbf{j}} e^{i \mathbf{q} \cdot \mathbf{j}} O_{\mathbf{j}, \mathbf{e}}$ and

$$
O_{\mathbf{j}, \mathbf{e}}=D_{\mathbf{j}, \mathbf{e}^{f}}^{\dagger} \frac{1}{\omega_{i}-\mathcal{H}+1 \Gamma} D_{\mathbf{j}, \mathbf{e}^{i}}
$$

which describes the evolution of the system in the RIXS experiment from the initial state to the final state via the intermediate states accessible via the core-hole to valence-band dipole transitions. $N$ is the number of lattice sites in the system. The dipole transition operator $D_{\mathbf{j}, \mathbf{e}}=$ $\sum_{\sigma, \alpha, \beta}\left(A_{\alpha}^{\mathbf{e}} p_{\mathbf{j} \alpha \sigma}^{\dagger} d_{\mathbf{j} \sigma}+\right.$ H.c. $)$ with $p_{\mathbf{j} \alpha \sigma}\left(d_{\mathbf{j} \sigma}\right)$ annihilates a hole in the $2 p(3 d)$ shell with spin $\sigma . A_{\alpha}^{\mathrm{e}}$ is the matrix element of the dipole transition between the $2 p_{\alpha}$ orbitals and the $3 d_{x^{2}-y^{2}}$ orbital written as $A_{\alpha}^{\mathbf{e}}=\left\langle d_{x^{2}-y^{2}, \sigma}|\hat{\epsilon} \cdot \hat{r}| p_{\alpha \sigma}\right\rangle$ for polarization $\hat{\epsilon}$. $\Gamma$ is the inverse core-hole lifetime. Note that with the exception of the schematic in Fig. 1, "hole notation" has been used throughout the paper, such that the dipole transitions at the $\mathrm{Cu} L$ edge correspond to transitions from the initial $3 d^{* 1} 2 p^{0}$ state to a $3 d^{* 0} 2 p^{1}$ intermediate state, and finally from the intermediate state to the final-state configuration. Here, $3 d^{*}$ corresponds to holes nominally (Zhang-Rice singlets [39]) in the single-band notation.

Here, we use the single-band Hubbard model as it carries the key features of correlated materials in the low-energy regime, which is the relevant energy regime for the study of charge and spin excitations for cuprates in RIXS measurements. The single-band results can be applied directly to cuprates and can be generalized to other multiorbital correlated materials. The Hamiltonian $\mathcal{H}$ defined on a 2D square lattice describes the relevant interactions of the "effective" $3 d$ and $2 p$ orbitals, consisting of two parts:

$$
\begin{gathered}
\mathcal{H}=H+H_{c}, \\
H=-t \sum_{\langle\mathbf{i}, \mathbf{j}\rangle, \sigma} d_{\mathbf{i} \sigma}^{\dagger} d_{\mathbf{j} \sigma}-t^{\prime} \sum_{\langle\langle\mathbf{i}, \mathbf{j}\rangle\rangle, \sigma} d_{\mathbf{i} \sigma}^{\dagger} d_{\mathbf{j} \sigma}+U \sum_{\mathbf{i}} n_{\mathbf{i} \uparrow}^{d} n_{\mathbf{i} \downarrow}^{d}, \\
H_{c}=\left(\epsilon^{d}-\epsilon^{p}\right) \sum_{\mathbf{i} \alpha \sigma} n_{\mathbf{i} \alpha \sigma}^{p}+U_{c} \sum_{\mathbf{i} \alpha \sigma \sigma^{\prime}} n_{\mathbf{i} \sigma}^{d} n_{\mathbf{i} \alpha \sigma^{\prime}}^{p} \\
+\lambda \sum_{\mathbf{i} \alpha \alpha^{\prime} \sigma \sigma^{\prime}} p_{\mathbf{i} \alpha \sigma}^{\dagger} \chi_{\alpha \alpha^{\prime}}^{\sigma \sigma^{\prime}} p_{\mathbf{i} \alpha^{\prime} \sigma^{\prime}} .
\end{gathered}
$$

The first part, Eq. (4), is the well-known single-band Hubbard model with the nearest (next-nearest) neighbor hopping $t\left(t^{\prime}\right)$ and on-site Hubbard repulsion $U$. Here, the operator $d^{\dagger}$ in the single-band Hubbard model creates a " $3 d^{*}$ " hole, which should be distinguished from the actual $\mathrm{Cu} 3 d_{x^{2}-y^{2}}$ hole in the multiband model. The second part, Eq. (5), describes (i) the energy splitting between the $2 p$ and the $3 d$ shells through the difference in site energy $\epsilon^{d}-\epsilon^{p}$, (ii) the repulsion between the $2 p$ and the $3 d$ holes

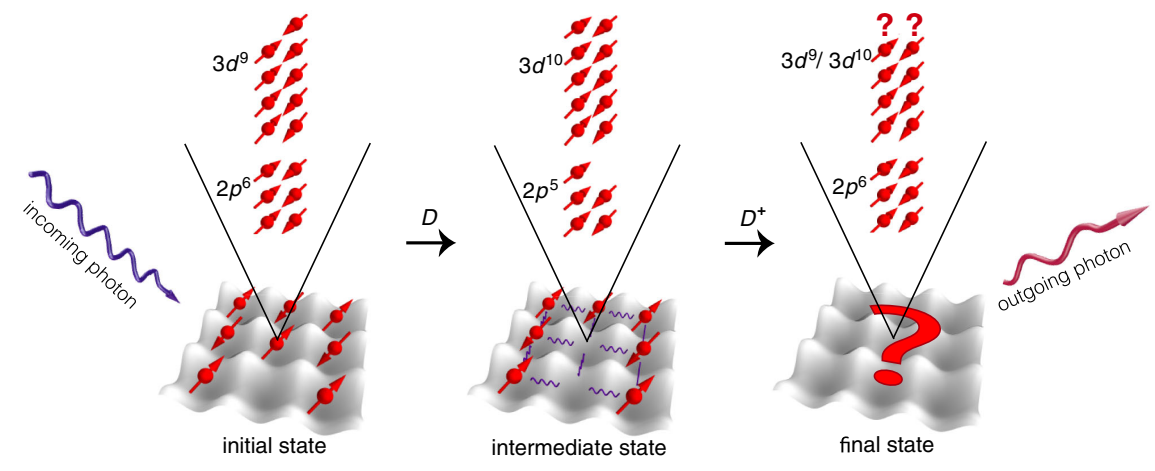

FIG. 1. A schematic that illustrates the RIXS process at the $\mathrm{Cu} L$-edge appropriate for cuprates. 
(the "core-hole potential") $U_{c}$, and (iii) the spin-orbit coupling in the $2 p$ shell $\lambda$ with the matrix elements $\chi_{\alpha \alpha^{\prime}}^{\sigma \sigma^{\prime}} \equiv\left\langle p_{\alpha \sigma}|\mathbf{I} \cdot \mathbf{s}| p_{\alpha^{\prime} \sigma^{\prime}}\right\rangle$, where the $\mathbf{I} \cdot \mathbf{s}$ term represents the spin-orbit coupling operator.

The RIXS cross section is calculated using exact diagonalization on a 12-site cluster. In this cluster, momentum points $(2 \pi / 3,0)$ and $(\pi / 2, \pi / 2)$ are accessible, providing information along both the Brillouin zone axis and diagonal, relevant for RIXS experiments. As the $\mathrm{Cu} 2 p$ core levels and the spin-orbit coupling are included (which also means that the total spin is not a good quantum number in the intermediate state), the Hilbert space size is about $10^{7}$. Ground-state eigenvectors and eigenvalues were obtained using the implicitly restarted Arnoldi method encoded in the Parallel ARPACK [40] libraries. The cross section itself was obtained using the biconjugate gradient stabilized method [41] and continued fraction expansion [42]. The numerical technique has been applied previously to calculate RIXS at the $\mathrm{Cu} K$ and $L$ edges [24,25]. Numerical results were obtained for parameters that can reproduce the low-energy physics for cuprates relatively well: $\epsilon^{d}-\epsilon^{p}=2325 t$ (which gives the typical splitting between the $\mathrm{Cu} 2 p$ and the $\mathrm{Cu} 3 d$ shell of $930 \mathrm{eV}$ if $t=0.4 \mathrm{eV}), U_{c}=4 t, \lambda=32.5 t, \Gamma=t, U=8 t, t^{\prime}=0.3 t$ (a typical value of the next-nearest-neighbor hopping in hole language). In this paper, all RIXS spectra are calculated at the $\mathrm{Cu} L_{3}$ resonance and at the $\mathrm{Cu}$ sites occupied by a single hole in the $3 d$ orbital for the ground state: The peak in the x-ray absorption spectroscopy (XAS) spectrum to which the RIXS resonance corresponds is shown in Fig. 7 in Appendix C. For doped systems, we investigate the resonance where the character of the intermediate state is similar to that in the undoped case on the core-hole site. The angle between the incident and the scattered photons is set to $50^{\circ}$, with the scattering plane parallel to $x z$, i.e., perpendicular to the $x y$ plane on which we define the $2 \mathrm{D}$ Hubbard Hamiltonian, and the incoming polarization is chosen to be $\pi$. This scattering geometry is consistent with that used most commonly in RIXS measurements for cuprates [9-11,13-16]. The relation between polarization and the transferred momenta $[27,30,31,43]$ follows from this scattering geometry: $\mathbf{e}^{i}=(\sin \theta, 0, \cos \theta)$, $\mathbf{e}^{f}=\left[-\cos \left(\theta-40^{\circ}\right), 0, \sin \left(\theta-40^{\circ}\right)\right] \quad\left[\mathbf{e}^{f}=(0,-1,0)\right]$ for outgoing $\pi(\sigma)$ polarization, $\theta \in\left[0^{\circ}, 130^{\circ}\right]$, and the angle $\theta$ is related to the transferred momentum via $k_{x}=1.07 \pi \sin \left(\theta-65^{\circ}\right)$. The calculated RIXS spectra will be presented in Figs. 2-5. The comparison with the approximated spectra will be presented in Sec. II C below.

\section{B. Approximate cross section}

The approximations follow from integrating out the corehole degrees of freedom using two approaches. We first assume that the energy of the incoming photon $\omega_{i}$ is tuned
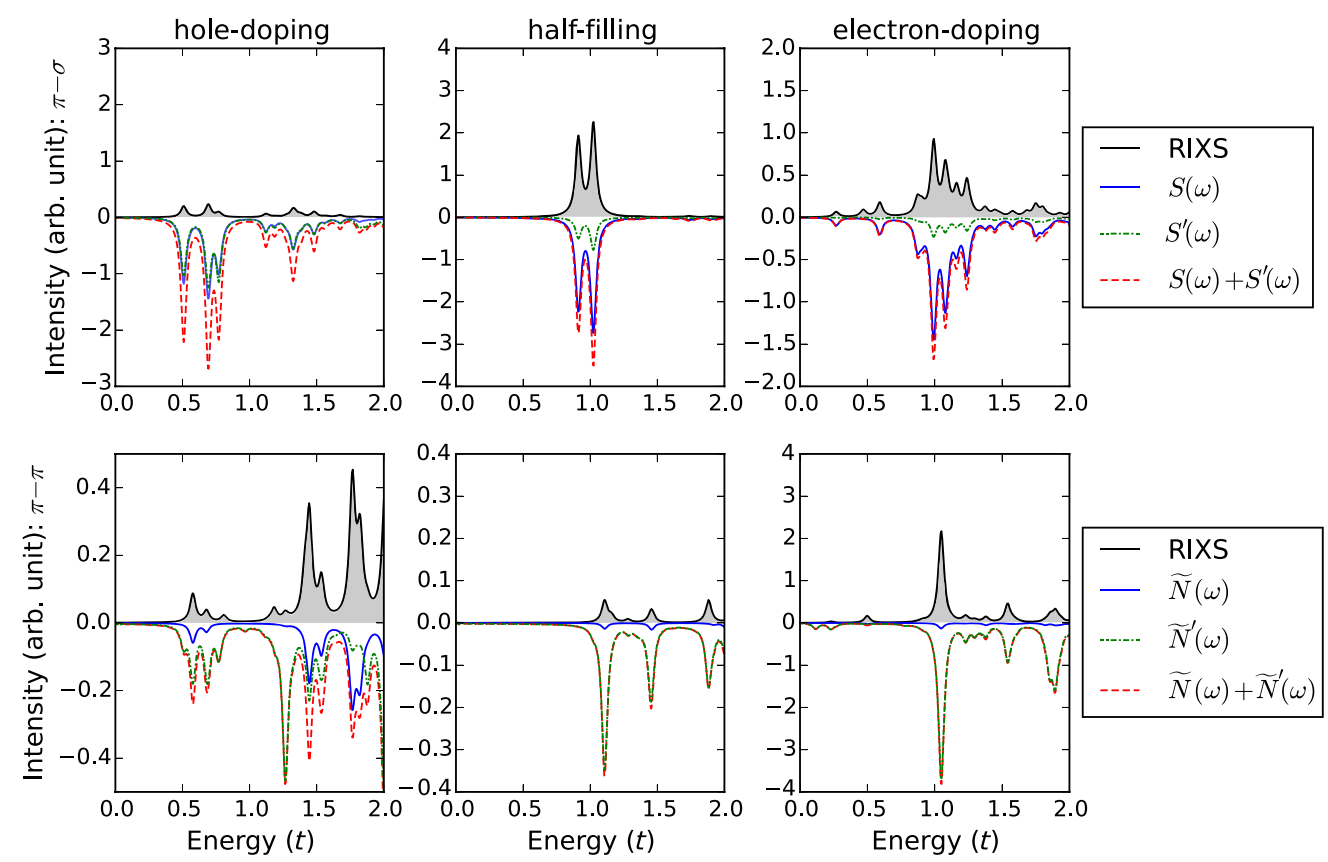

FIG. 2. Exact RIXS cross sections and approximations calculated using exact diagonalization. Top (bottom) panels show spectra for the $\pi-\sigma(\pi-\pi)$ polarization geometries. Left, middle, and right panels show RIXS spectra calculated for $n=0.83$ ("hole-doping"), $n=1$ ("half-filling"), and $n=1.17$ ("electron-doping") electron fillings, respectively. The spectra are summed over all momenta along the nodal direction and along the $k_{y}=0$ direction, which are accessible in RIXS on a 12-site cluster, weighted with the RIXS form factors, i.e., $S(\omega)=\sum_{\mathbf{q}}\left|W_{\pi-\sigma}\right|^{2} S(\mathbf{q}, \omega), \tilde{N}(\omega)=\sum_{\mathbf{q}}\left|W_{\pi-\pi}\right|^{2} \tilde{N}(\mathbf{q}, \omega)$ and similarly for $S^{\prime}(\omega)$ and $\tilde{N}^{\prime}(\omega)$. The elastic response has been removed in each panel. 

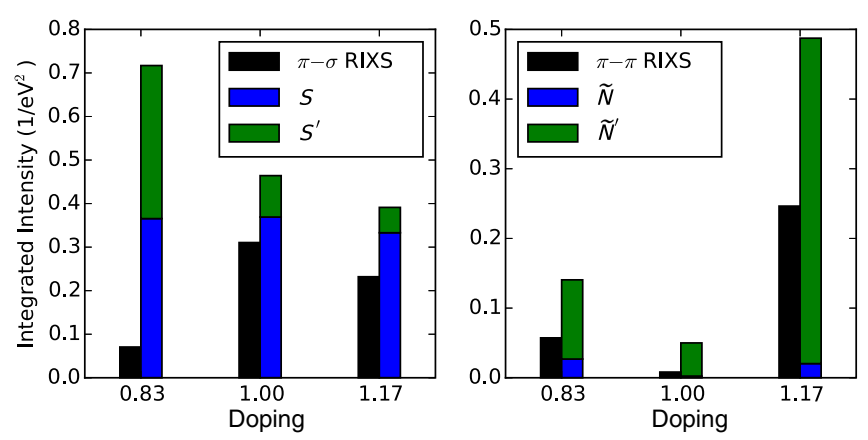

FIG. 3. Momentum and energy integrated spectral weight for full RIXS $\int_{0}^{1.6 t} I(\omega) d \omega$ and the various approximations $S=\int_{0}^{1.6 t} S(\omega) d \omega, \tilde{N}=\int_{0}^{1.6 t} \tilde{N}(\omega) d \omega, S^{\prime}=\int_{0}^{1.6 t} S^{\prime}(\omega) d \omega, \tilde{N}^{\prime}=$ $\int_{0}^{1.6 t} \tilde{N}^{\prime}(\omega) d \omega$ with $S(\omega), \tilde{N}(\omega), S^{\prime}(\omega), \tilde{N}^{\prime}(\omega)$ defined as in the caption of Fig. 2, RIXS spectra. The $\pi-\sigma(\pi-\pi)$ relative polarizations are shown on the left (right) panels, respectively. Note that the spectral weight $\tilde{N}=5 \times 10^{-5}(\mathrm{eV})^{-2}$ in the halffilled case does not appear in the figure. All momenta along the nodal direction and along the $q_{y}=0$ direction accessible on a 12-site cluster are taken into account (elastic response excluded).

to the main resonance at the $\mathrm{Cu} L_{3}$ edge, as dictated by $H_{c}$ [44]. This is equivalent to a projection into a subspace with only one $3 d^{*}$ hole on the core-hole site in either the initial state $|i\rangle$ or the final state $|f\rangle$. We then perform the UCL approximation by expanding the RIXS operator, Eq. (2), in a power series in $\mathcal{H} / \Gamma$ and only keeping the first two terms. See Appendix A for details. These approximations can be written separately for the two polarization conditions as follows:

$\pi-\sigma$ RIXS in the UCL approximation:

$$
\begin{gathered}
I_{\pi-\sigma}^{\mathrm{UCL}}(\mathbf{q}, \omega)=\left|W_{\pi-\sigma}\right|^{2}\left\{S(\mathbf{q}, \omega)+S^{\prime}(\mathbf{q}, \omega)\right\}, \\
S(\mathbf{q}, \omega)=\sum_{f}\left|\left\langle f\left|S_{\mathbf{q}}^{z}\right| i\right\rangle\right|^{2} \delta\left(\omega+E_{i}-E_{f}\right), \\
S^{\prime}(\mathbf{q}, \omega)=\frac{z^{2} t^{2}}{N^{2} \Gamma^{2}} \sum_{f} \mid\langle f| \sum_{\mathbf{k}, \mathbf{k}^{\prime}} \varepsilon_{-\mathbf{k}^{\prime}+\mathbf{k}+\mathbf{q}} S_{\mathbf{k}}^{z \prime} \\
\times\left. d_{\mathbf{k}, \sigma}^{\dagger} d_{-\mathbf{k}^{\prime}+\mathbf{k}+\mathbf{q}, \sigma}|i\rangle\right|^{2} \delta\left(\omega+E_{i}-E_{f}\right),
\end{gathered}
$$

where the local RIXS form factor $W_{\pi-\sigma}=-12 \sin \theta /(3 \Gamma)$, the spin operator $S_{\mathbf{q}}^{z}=1 /(2 \sqrt{N}) \sum_{\mathbf{k}}\left(d_{\mathbf{k}, \uparrow}^{\dagger} d_{\mathbf{q}+\mathbf{k}, \uparrow}-\right.$ $\left.d_{\mathbf{k}, \downarrow}^{\dagger} d_{\mathbf{q}+\mathbf{k}, \downarrow}\right), z=4$ is the $2 \mathrm{D}$ coordination number, and the 2D structure factor is $\varepsilon_{\mathbf{k}}=\gamma_{\mathbf{k}}+t^{\prime} \eta_{\mathbf{k}} / t$, with $\gamma_{\mathbf{k}}=$ $\left(\cos k_{x}+\cos k_{y}\right) / 2$ and $\eta_{\mathbf{k}}=\cos k_{x} \cos k_{y}$. The structure factor $\varepsilon_{\mathbf{k}}$ has $A_{1 g}$ symmetry. Note that the first term of the expansion $S(\mathbf{q}, \omega)$ has the form of the spin dynamical structure factor, while the second term $S^{\prime}(\mathbf{q}, \omega)$ is a rather complicated four-particle response that probes spin and charge excitations. The latter corresponds to the three-spin Green's function (see Appendix B) and to joint spin and charge excitations.

\section{$\pi-\pi$ RIXS in UCL approximation:}

$$
\begin{aligned}
& I_{\pi-\pi}^{\mathrm{UCL}}(\mathbf{q}, \omega)=\left|W_{\pi-\pi}\right|^{2}\left\{\tilde{N}(\mathbf{q}, \omega)+\tilde{N}^{\prime}(\mathbf{q}, \omega)\right\}, \\
& \tilde{N}(\mathbf{q}, \omega)=\sum_{f}\left|\left\langle f\left|\tilde{n}_{\mathbf{q}}\right| i\right\rangle\right|^{2} \delta\left(\omega+E_{i}-E_{f}\right), \\
& \tilde{N}^{\prime}(\mathbf{q}, \omega)= \frac{z^{2} t^{2}}{N \Gamma^{2}} \sum_{f}\left|\left\langle f\left|\sum_{\mathbf{k}} \varepsilon_{\mathbf{q}+\mathbf{k}} \tilde{d}_{\mathbf{k}, \sigma}^{\dagger} d_{\mathbf{q}+\mathbf{k}, \sigma}\right| i\right\rangle\right|^{2} \\
& \times \delta\left(\omega+E_{i}-E_{f}\right),
\end{aligned}
$$

where the constrained density operator is $\tilde{n}_{\mathbf{i}}=\sum_{\sigma} \tilde{d}_{\mathbf{i} \sigma}^{\dagger} \tilde{d}_{\mathbf{i} \sigma}$ with constrained fermions $\tilde{d}_{\mathbf{i} \sigma}^{\dagger}=d_{\mathbf{i} \sigma}^{\dagger}\left(1-n_{\mathbf{i} \sigma}\right)$ and $\tilde{d}_{\mathbf{i} \sigma}=\left(1-n_{\mathbf{i} \bar{\sigma}}\right) d_{\mathbf{i} \sigma}$, and the local RIXS form factor $W_{\pi-\pi}=-2 \sin \theta \cos \left(\theta-40^{\circ}\right) /(3 \Gamma)$.

Before evaluating the correlation functions, we want to highlight that none of these approximations gives the standard charge dynamical structure factors for the parallel-polarization channel. The first term of the expansion, $\tilde{N}(\mathbf{q}, \omega)$, is not the standard charge dynamical structure factor. It represents a more complicated four-particle response function in the original space of unprojected fermions. In the following, it carries information about projected charge excitations, which should in no way be confused with information about the full charge response. That RIXS does not probe the standard charge response stems from the fact that initial states with double occupancy on the core-hole site cannot be excited in the RIXS process because of the Pauli principle. The second term $\tilde{N}^{\prime}(\mathbf{q}, \omega)$, a complicated four-particle response function as well, corresponds to two-spin or bimagnon excitations (see Appendix B) and other symmetry-projected charge excitations, represented again by a correlator beyond the familiar two-particle charge response.

\section{Comparing exact and approximate results}

In the following, we present a systematic comparison of the full RIXS spectra with the approximations using the UCL expansion for the two polarization conditions. As a momentum-resolved technique, RIXS has the power to measure the dispersion of elementary excitations, which is one of its main advantages compared to traditional optical or Raman scattering, where the momentum transfer is limited to $q \sim 0$ [23]. However, there are limitations in cluster size, as well as a limited number of poles using a finite-size cluster. Thus, summing over all the accessible momentum points (results as shown in Fig. 2) gives us a complete picture of the energies and the distribution of intensities for the excitations, so that the comparisons to the approximations can be made in a single shot. To better quantify this comparison, we also calculate and compare the total spectral weight carried by the excitations, cf. Fig. 3. Nevertheless, in Sec. II D, we compare exact RIXS cross sections and approximations at the momentum 
points $(2 \pi / 3,0)$ and $(\pi / 2, \pi / 2)$ and discuss connections to experiments.

The full cross sections are shown in Fig. 2 for two polarization channels: (i) the cross-polarized channel [ $\pi-\sigma$ with $\pi(\sigma)$ incoming (outgoing) polarization] and (ii) the parallel-polarized channel $[\pi-\pi$ with $\pi(\pi)$ incoming (outgoing) polarization]. For each channel, the spectra are calculated for three different doping levels $(n=0.83$, hole doping, $n=1$, half filling, and $n=1.17$, electron doping). The $\pi-\sigma$ RIXS spectra presented here agree with those presented in Ref. [24]. The approximate cross sections $S(\omega)$ and $S^{\prime}(\omega)$ for cross polarization and $\tilde{N}(\omega)$ and $\tilde{N}^{\prime}(\omega)$ for parallel polarization are also shown in Fig. 2. The results for both full and approximated RIXS are shown for a Lorentzian broadening with half width at half maximum $(\mathrm{HWHM})=0.025 t$ for the energy transfer. Note that the spectra in Fig. 2 correspond to a momentum summation over all points accessible in the 12-site cluster, to provide a holistic picture of the character of excitations probed by RIXS and the utility of various approximations (see caption of Fig. 2 for details).

First note the results in the cross-polarized channel (the $\pi-\sigma$ channel). On a qualitative level, the line shape of the full RIXS cross section can be reproduced well by the spin dynamical structure factor $S(\mathbf{q}, \omega)$ (the first term of the UCL approximation). This is true at half filling, where all charge excitations have been gapped out, while in either the electronor hole-doped cases, one can observe some relatively small discrepancies between the two spectra. When adding higherorder terms from the effective expansion, to a large extent this observation remains unchanged since these terms encode similar excitations to $S(\mathbf{q}, \omega)$ together with excitations of mixed charge and spin character, as can be seen readily from the form of the operator in Eq. (8).

However, on a quantitative level, this comparison breaks down, with discrepancies in the overall intensity and integrated spectral weight which can become very large (see Fig. 3). While one may have expected that higher-order terms in the effective expansion should provide a more satisfactory qualitative and quantitative agreement, they do not help in reducing the differences; on the contrary, these additional terms actually enhance the quantitative mismatch. Note that the first two terms of the UCL expansion suggest larger spectral weight for the hole-doped case compared to that with electron doping, in contrast to the behavior for the RIXS cross section. This suggests that the differences cannot be attributed to a simple rescaling factor.

The quantitative mismatch between RIXS at the $\mathrm{Cu} L$ edge and the approximations highlights the role that the intermediate-state wave function plays in the RIXS spectra, just as in the case for RIXS at the $\mathrm{Cu} K$ edge [25]. RIXS is an intrinsic four-particle process, where the wavefunction overlaps between the ground state and intermediate states and between intermediate states and final states both play an important role. Neglecting the details of the intermediate-state wavefunction might still provide information on the fundamental excitation energies, but unfortunately, it cannot provide reasonable spectral weights on a quantitative level. For the discussions in terms of the role of these intermediate states in XAS, see Appendix C. That being said, both numerically and empirically, in the crosspolarized scattering geometry, the RIXS cross section qualitatively corresponds to the spin dynamical structure factor, which encodes information about spin excitations at the two-particle level, underscoring RIXS utility as a complementary probe to inelastic neutron scattering.

An altogether different situation arises in the parallelpolarized channel (the $\pi-\pi$ channel). While a comparison between the RIXS spectrum and the "projected" charge excitations produces a modest qualitative agreement between the line shapes, both missing peaks and significant differences in the spectral weights undermine any quantitative value in this comparison. The addition of the higher-order terms seems to be needed for a better qualitative comparison of the line shapes, although both terms support similar spectral excitations based on the form of the operators in Eqs. (10) and (11), and a spectral weight analysis precludes any quantitative agreement. In both cases, while the RIXS spectral line shape may be approximated by the two expansion terms, neither provides a faithful representation for the proper two-particle charge response encoded in the simple dynamical structure factor, placing statements about the true charge excitation character of the RIXS cross section on less solid footing. Similar analysis with different values of core-hole potential $U_{c}$ leads to similar conclusions (see Appendix D).

\section{Consequences for RIXS experiments}

The preceding section presented a comparison between cross sections integrated in momentum, as well as energy for a total spectral weight analysis. In this section, we show spectra at two particular momenta: $\mathbf{q}=(2 \pi / 3,0)$ and $\mathbf{q}=(\pi / 2, \pi / 2)$ (see Figs. 4 and 5 ) to underscore those results, shown in a context amenable to experiment. When doped, the Hubbard model, and by extension cuprates, will possess spin and charge excitations in a similar lowenergy regime which will appear, either directly or in a more complicated way reflecting the complexity of the cross section, in the RIXS spectrum for the crossed- and parallel-polarization channels, respectively. Thus, to satisfactorily distinguish between the magnetic and charge channels, or two-spin excitations, one must perform measurements that can discriminate the outgoing polarizations. Unfortunately, to this point, RIXS experiments have been unable to fully distinguish between the cross-polarized and parallel-polarized channels, making some statements with the help of a careful analysis of experimental RIXS scattering geometry. The newly constructed, state-of-the-art RIXS end station at ESRF now provides an opportunity to perform such 

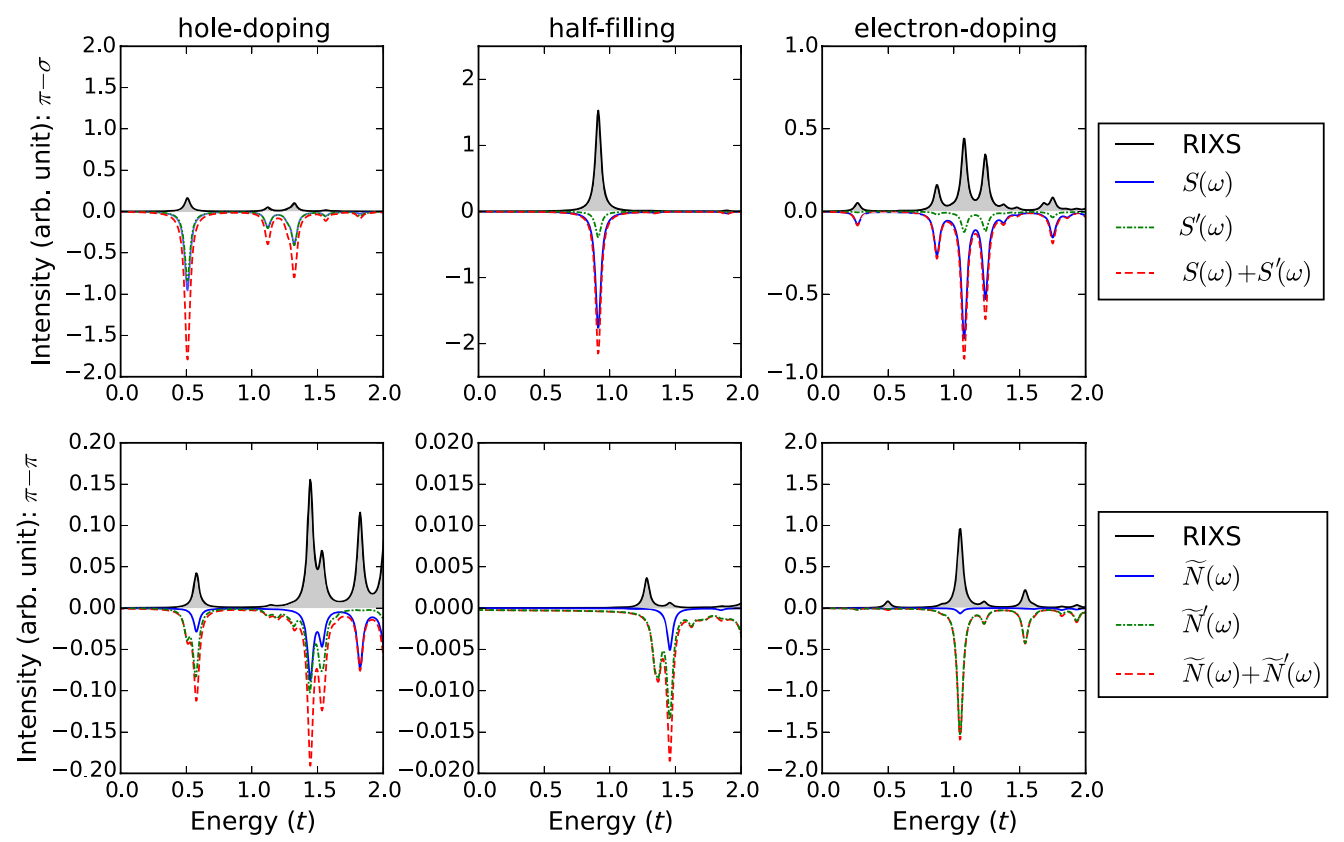

FIG. 4. Cross sections at $\mathbf{q}=(\mathbf{2} \boldsymbol{\pi} / \mathbf{3}, \mathbf{0})$ with the elastic response removed. Top (bottom) panels show spectra for the $\pi-\sigma$ ( $\pi-\pi)$ polarization. Left, middle, and right panels show RIXS spectra for $n=0.83, n=1$, and $n=1.17$ electron fillings, respectively. The approximate spectra are weighted with the RIXS form factors, i.e., $S(\mathbf{q}, \omega) \rightarrow\left|W_{\pi-\sigma}\right|^{2} S(\mathbf{q}, \omega), \tilde{N}(\mathbf{q}, \omega) \rightarrow\left|W_{\pi-\pi}\right|^{2} \tilde{N}(\mathbf{q}, \omega)$ and similarly for $S^{\prime}(\mathbf{q}, \omega)$ and $\tilde{N}^{\prime}(\mathbf{q}, \omega)$.

measurements (cf. Ref. [17]); other end stations (currently operational or to be commissioned in the coming years) also would allow for the differentiation between the crossed- and parallel-polarized channels.
Even with outgoing polarization discrimination, in either the crossed- or parallel-polarized channels, one needs to carefully invoke either $S(\mathbf{q}, \omega)$ or $\tilde{N}(\mathbf{q}, \omega)$ as approximations for the full RIXS cross section. This especially may be
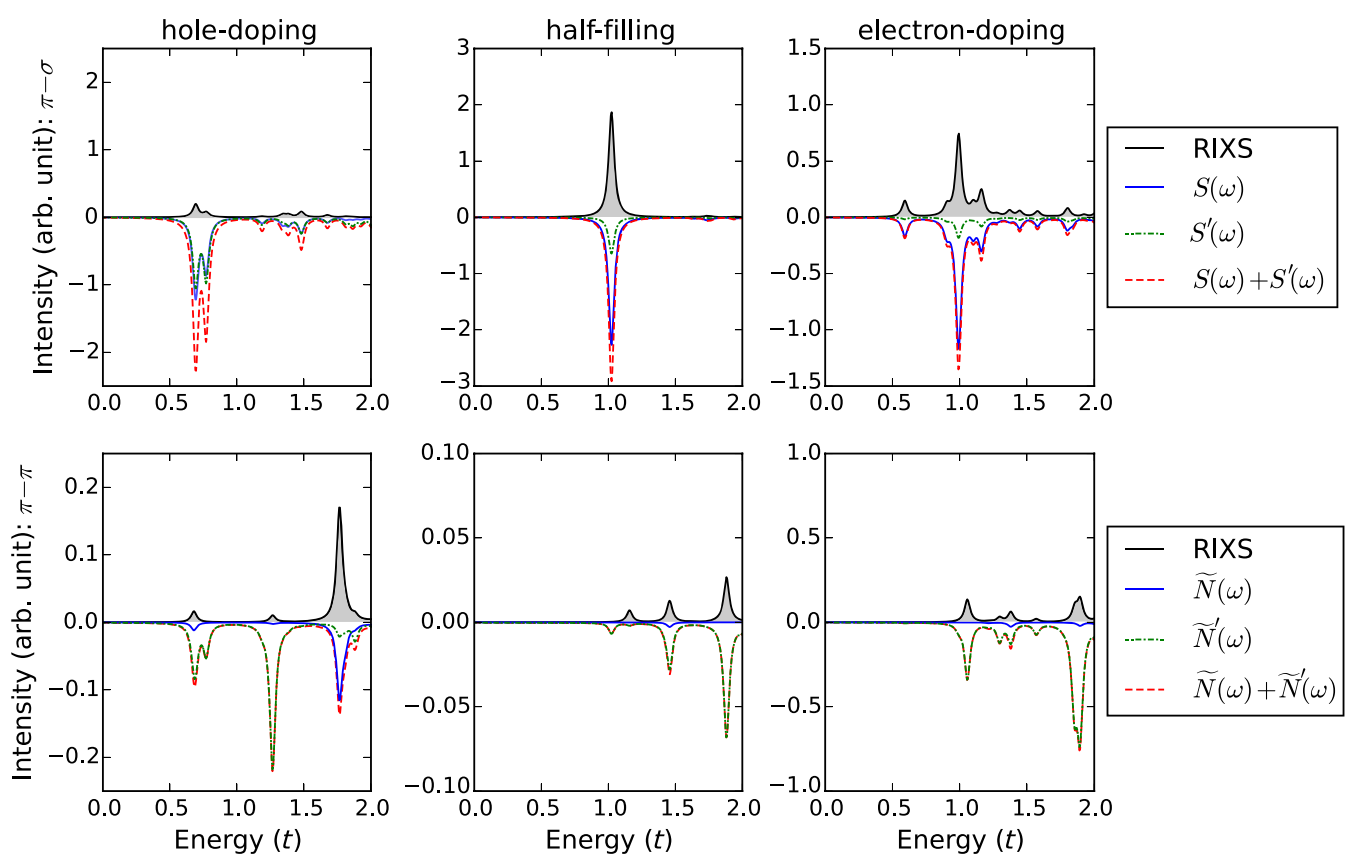

FIG. 5. Cross sections at the $\mathbf{q}=(\pi / 2, \pi / 2)$ point with the elastic response removed. Top (bottom) panels show spectra for the $\pi-\sigma$ $(\pi-\pi)$ polarization. Left, middle, and right panels show RIXS spectra calculated for $n=0.83, n=1$, and $n=1.17$ electron fillings, respectively. The approximate spectra are weighted with the RIXS form factors, i.e., $S(\mathbf{q}, \omega) \rightarrow\left|W_{\pi-\sigma}\right|^{2} S(\mathbf{q}, \omega), \tilde{N}(\mathbf{q}, \omega) \rightarrow$ $\left|W_{\pi-\pi}\right|^{2} \tilde{N}(\mathbf{q}, \omega)$ and similarly for $S^{\prime}(\mathbf{q}, \omega)$ and $\tilde{N}^{\prime}(\mathbf{q}, \omega)$. 
true when analyzing RIXS spectral weights as a function of doping. None of these approximations addresses intermediate-state effects or, equivalently, differences in the cross section with changes in the incoming photon energy [25]. Thus, to address the full resonant profile, one always needs to calculate the full RIXS cross section.

\section{CONCLUSIONS AND DISCUSSIONS}

We have performed small-cluster exact diagonalization calculations using the single-band Hubbard model plus the $\mathrm{Cu} 2 p$ core, to study the RIXS $L$-edge cross sections using the Kramers-Heisenberg formula [Eqs. (1) and (2)], and we compare this with the approximate RIXS cross section obtained from the UCL expansion. The results show that the UCL approximation cannot always reproduce the full RIXS cross section. In other words, one must carefully apply approximations for calculating the RIXS cross section. The nonlocal character of the intermediate state can become particularly important in correlated ground states with longer-range entanglement. Therefore, we suggest the full RIXS simulations will be needed to verify the character of excitations.

In the cross-polarized channel, we have shown that on a qualitative level, $\mathrm{Cu} L$-edge RIXS line shapes correspond to the spin dynamical structure factor $S(\mathbf{q}, \omega)$, consistent with lowest-order approximations as postulated by the fast collision approximation (or the effective operator approach) [24,27,31,32] (see Appendix E). As a consequence, we expect that the line shapes reported from cross-polarized RIXS experiments can be reproduced to some extent by theoretical modeling of the spin dynamical structure factors (or empirically through inelastic neutron scattering experiments when also considering differences in the effective matrix elements between the two techniques). However, the detailed analysis in this paper suggests that a quantitative comparison between RIXS and the two-particle spin and charge dynamical structure factors would be impractical. One should not expect a meaningful comparison between different spectral weights obtained from these different techniques, either experimentally or from simulation.

In the parallel-polarized channel, the situation is further complicated by the operator form taken by the approximations themselves. On a qualitative level, the primary contributions seem to follow from higher-order terms $\tilde{N}^{\prime}(\mathbf{q}, \omega)$, with a notable exception at half filling (see Appendix E). However, any precise quantitative comparison to experiment would require calculating the full RIXS cross section. At the same time, none of the terms in the approximations corresponds to proper two-particle charge excitations, but rather, they inherently reflect the complexity of the RIXS process. Thus, while line shapes in RIXS should closely resemble the line shapes of the (projected) charge excitations, the spectral weights may be quite different, with a more complicated analysis required to tease out the character of various spectral peaks.
In modeling the RIXS spectra for strongly correlated materials, we use the single-band Hubbard model, which is a simple model that possesses the key features of correlated materials relevant to charge and spin excitations in the low-energy regime. As the key physics for the singleband Hubbard and the multiband Hubbard models remain similar, we expect that the conclusion of our study can be generalized to a multiband Hubbard system: RIXS spectra in the cross-polarized channel may connect to the spin dynamical structure factor, but the full description of RIXS goes beyond the simple two-particle response function.

Besides cuprates, RIXS has also been utilized to measure excitations in iron-pnictide superconductors [45,46], $f$ electron systems [47], and many other materials [48-50], including out of equilibrium [51]. Indeed, the idea of using polarization control to separate the spin-flip and non-spinflip excitations can be generalized to all kinds of materials. When the incoming and outgoing photon polarizations are perpendicular, because of angular-momentum (spin) conservation, RIXS qualitatively measures spin-flip excitations regardless of the details of the microscopic Hamiltonian. This makes RIXS a powerful technique for separately measuring the spin and charge excitations.

\section{ACKNOWLEDGMENTS}

We acknowledge support from the DOE-BES Division of Materials Sciences and Engineering (DMSE) under Contract No. DE-AC02-76SF00515 (Stanford/SIMES). K. W. acknowledges support from the Polish National Science Center (NCN) under Project No. 2012/04/A/ ST3/00331. We are grateful for insightful discussions with L. Braicovich, J. van den Brink, I. Eremin, G. Ghiringhelli, D. J. Huang, B. J. Kim, A. M. Oleś, and G. A. Sawatzky. A portion of the computational work was performed using the resources of the National Energy Research Scientific Computing Center (NERSC) supported by the U.S. Department of Energy, Office of Science, under Contract No. DE-AC02-05CH11231.

C. J. J. and K. W. contributed equally to this work.

\section{APPENDIX A: DERIVATION OF THE UCL APPROXIMATION FOR RIXS AT THE CU $L$ EDGE}

The UCL approximation should be valid when all the relevant eigenenergies of the intermediate-state Hamiltonian $\mathcal{H}$ are much smaller than the inverse core-hole lifetime $\Gamma$. We use the following spectral decomposition:

$$
\frac{1}{\omega_{i}-\mathcal{H}+i \Gamma}=\sum_{|N\rangle}|N\rangle\langle N| \frac{1}{\omega_{i}-\mathcal{E}_{N}+i \Gamma},
$$

where $\{|N\rangle\}$ are eigenstates of $\mathcal{H}$ with energy $\left\{\mathcal{E}_{N}\right\}$. We are interested in RIXS at the resonant edge between the $2 p^{0} 3 d^{* 1}$ initial configuration and the $2 p^{1} 3 d^{* 0}$ intermediate-state 
configuration. (All expressions are presented in hole language.) This means that we need to exclude all intermediate states $|N\rangle$ that contain one hole in the $d$ orbital on site $\mathbf{j}$, i.e., on the core-hole site. We add a projection operator $\tilde{P}_{\mathbf{j}}$ and rewrite the above expression as

$$
\sum_{|N\rangle}|N\rangle\left\langle N\left|\frac{1}{\omega_{i}-\mathcal{E}_{N}+i \Gamma} \rightarrow \sum_{|N\rangle} \tilde{P}_{\mathbf{j}}\right| N\right\rangle\langle N| \tilde{P}_{\mathbf{j}} \frac{1}{\omega_{i}-\mathcal{E}_{N}+i \Gamma} .
$$

We define the following Hamiltonians $\bar{H}=H+$ $U_{c} \sum_{\mathbf{i} \alpha \sigma \sigma^{\prime}} n_{\mathbf{i} \sigma}^{d} n_{\mathbf{i} \alpha \sigma^{\prime}}^{p}$ and $\bar{H}_{c}=H_{c}-U_{c} \sum_{\mathbf{i} \alpha \sigma \sigma^{\prime}} n_{\mathbf{i} \sigma}^{d} n_{\mathbf{i} \alpha \sigma^{\prime}}^{p}$. Note that $\bar{H}$ and $\bar{H}_{c}$ commute (and $\mathcal{H}=\bar{H}+\bar{H}_{c}$ ), since the intermediate states of RIXS are such that they always contain either a hole in the $2 p$ shell or in the $3 d$ shell (guaranteed by the projection operators $\tilde{P}_{\mathbf{j}}$ ). Then, we obtain

$$
|N\rangle=|n\rangle\left|n_{c}\right\rangle \quad \mathcal{E}_{N}=\varepsilon_{n}+\varepsilon_{n_{c}},
$$

where $|n\rangle$ are eigenstates of $\bar{H}$ with energy $\varepsilon_{n}$, and $\left|n_{c}\right\rangle$ are eigenstates of $\bar{H}_{c}$ with energy $\varepsilon_{n_{c}}$. Consequently, we can write

$$
\begin{aligned}
\frac{1}{\omega_{i}-\mathcal{H}+i \Gamma}= & \sum_{|n\rangle,\left|n_{c}\right\rangle} \tilde{P}_{\mathbf{j}}|n\rangle\left\langle n \mid n_{c}\right\rangle\left\langle n_{c}\right| \tilde{P}_{\mathbf{j}} \\
& \times \frac{1}{\omega_{i}-\varepsilon_{n}-\varepsilon_{n_{c}}+i \Gamma} .
\end{aligned}
$$

Note that with a single hole in the $p$ shell, there are just two eigenstates of $\bar{H}_{c}: n_{c} \in\left|L_{2}\right\rangle,\left|L_{3}\right\rangle$ with energies $\varepsilon_{L_{2}}$, $\varepsilon_{L_{3}}$. They correspond to the two " $j$ " eigenstates $j=1 / 2$ and $j=3 / 2$, respectively, split by the spin-orbit coupling $\propto \lambda$. (We note that $j$ represents the angular momentum and $\mathbf{j}$ represents site $\mathbf{j}$ on the cluster.) This implies

$$
\begin{aligned}
& \frac{1}{\omega_{i}-\mathcal{H}+i \Gamma} \\
& =\sum_{|n\rangle} \tilde{P}_{\mathbf{j}}|n\rangle\left\langle n \mid L_{2}\right\rangle\left\langle L_{2}\right| \tilde{P}_{\mathbf{j}} \frac{1}{\omega_{i}-\varepsilon_{n}-\varepsilon_{L_{2}}+i \Gamma} \\
& \quad+\sum_{|n\rangle} \tilde{P}_{\mathbf{j}}|n\rangle\left\langle n \mid L_{3}\right\rangle\left\langle L_{3}\right| \tilde{P}_{\mathbf{j}} \frac{1}{\omega_{i}-\varepsilon_{n}-\varepsilon_{L_{3}}+i \Gamma} .
\end{aligned}
$$

Resonance approximation.-We assume that the incoming $\mathrm{x}$-ray photons are tuned to the $L_{3}$ resonance, i.e., $\omega_{i} \simeq \varepsilon_{L_{3}}$. Since $\lambda \gg \Gamma$ (and all eigenenergies $\varepsilon_{n} \ll \lambda$ ), we can neglect the contribution from the $L_{2}$ resonance and obtain

$\frac{1}{\omega_{i}-\mathcal{H}+i \Gamma}=\sum_{|n\rangle} \tilde{P}_{\mathbf{j}}|n\rangle\left\langle n \mid L_{3}\right\rangle\left\langle L_{3}\right| \tilde{P}_{\mathbf{j}} \frac{1}{-\varepsilon_{n}+i \Gamma}$.

UCL expansion.-We adopt the UCL expansion $[37,52,53]$ for $\mathrm{Cu} L$-edge RIXS to obtain

$$
\frac{1}{\omega_{i}-\mathcal{H}+i \Gamma}=\tilde{P}_{\mathbf{j}}\left|L_{3}\right\rangle\left\langle L_{3}\right| \sum_{l=0}^{+\infty} \frac{\bar{H}^{l}}{(i \Gamma)^{l+1}} \tilde{P}_{\mathbf{j}} .
$$

This means that the RIXS operator can be rewritten as

$$
O_{\mathbf{j}, \mathbf{e}}=\frac{1}{i \Gamma} D_{\mathbf{j}, \mathbf{e}}^{\dagger} \tilde{P}_{\mathbf{j}}\left|L_{3}\right\rangle\left\langle L_{3}\right| \sum_{l=0}^{+\infty} \frac{\bar{H}^{l}}{(i \Gamma)^{l}} \tilde{P}_{\mathbf{j}} D_{\mathbf{j}, \mathbf{e}^{i}} .
$$

Second-order UCL approximation.-Keeping only terms with $l=0$ and $l=1$ gives

$$
\begin{gathered}
O_{\mathbf{j}, \mathbf{e}}=O_{\mathbf{j}, \mathbf{e}}^{(1)}+O_{\mathbf{j}, \mathbf{e}}^{(2)}, \\
O_{\mathbf{j}, \mathbf{e}}^{(1)}=\frac{1}{i \Gamma} D_{\mathbf{j}, \mathbf{e}}^{\dagger} \tilde{P}_{\mathbf{j}}\left|L_{3}\right\rangle\left\langle L_{3}\right| \tilde{P}_{\mathbf{j}} D_{\mathbf{j}, \mathbf{e}^{i}}, \\
O_{\mathbf{j}, \mathbf{e}}^{(2)}=\frac{1}{(i \Gamma)^{2}} D_{\mathbf{j}, \mathrm{e}^{f}}^{\dagger} \tilde{P}_{\mathbf{j}}\left|L_{3}\right\rangle\left\langle L_{3}\right| \tilde{H}_{\mathbf{P}_{\mathbf{j}} D_{\mathbf{j}, \mathbf{e}^{i}}}
\end{gathered}
$$

The validity of this approximation has been discussed in detail in the main text of the paper.

Change of projection operators.- - It is convenient to use another operator, $P_{\mathbf{j}}$, which projects to the sector with no double occupancy in the $d$ level on site $\mathbf{j}$, i.e., where the core hole is created. This gives

$$
\tilde{P}_{\mathbf{j}} D_{\mathbf{j}, \mathbf{e}^{i}}=D_{\mathbf{j}, \mathbf{e}^{i}} P_{\mathbf{j}}
$$

A similar expression also holds for the $D_{\mathbf{j}, \mathrm{e}^{f}}^{\dagger}$ dipole operator.

The $l=1$ term. - Next, we evaluate

$$
P_{\mathbf{j}} D_{\mathbf{j}, \mathbf{e}^{f}}^{\dagger}\left|L_{3}\right\rangle\left\langle L_{3}\left|\bar{H} D_{\mathbf{j}, \mathbf{e}^{i}} P_{\mathbf{j}}\right| i\right\rangle .
$$

We note that

$$
P_{\mathbf{j}} D_{\mathbf{j}, \mathbf{e}^{f}}^{\dagger}\left|L_{3}\right\rangle\left\langle L_{3}\left|\bar{H}_{\mathbf{m} \mathbf{j}} D_{\mathbf{j}, \mathbf{e}^{i}} P_{\mathbf{j}}\right| i\right\rangle=0,
$$

where

$$
\begin{aligned}
\bar{H}_{\mathbf{m} \mathbf{j}}= & -t \sum_{m(j), \sigma}\left(d_{\mathbf{m} \sigma}^{\dagger} d_{\mathbf{j} \sigma}+\text { H.c. }\right)-t^{\prime} \sum_{\mathbf{m}^{\prime}(\mathbf{j}), \sigma}\left(d_{\mathbf{m}^{\prime} \sigma}^{\dagger} d_{\mathbf{j} \sigma}+\text { H.c. }\right) \\
& +U_{c} \sum_{\alpha \sigma \sigma^{\prime}} n_{\mathbf{j} \sigma}^{d} n_{\mathbf{j} \alpha \sigma^{\prime}}^{p}+U n_{\mathbf{j} \uparrow}^{d} n_{\mathbf{j} \downarrow}^{d},
\end{aligned}
$$

since all terms in the Hamiltonian that contain site $\mathbf{j}$ will vanish when "sandwiched" between the dipole operators $D$ and evaluated on the initial state $|i\rangle$. Here, $\mathbf{m}(\mathbf{j})$ and $\mathbf{m}^{\prime}(\mathbf{j})$ are nearest and next-nearest neighbors of site $\mathbf{j}$.

Thus, the following expression holds:

$$
\begin{aligned}
P_{\mathbf{j}} D_{\mathbf{j}, \mathbf{e}^{f}}^{\dagger}\left|L_{3}\right\rangle\left\langle L_{3}\left|\bar{H} D_{\mathbf{j}, \mathbf{e}^{i}} P_{\mathbf{j}}\right| i\right\rangle= & P_{\mathbf{j}} D_{\mathbf{j}, \mathbf{e}^{f}}^{\dagger}\left|L_{3}\right\rangle\left\langle L_{3}\right| \\
& \times\left(\bar{H}-\bar{H}_{\mathbf{m} \mathbf{j}}\right) D_{\mathbf{j}, \mathbf{e}^{\mathbf{e}}} P_{\mathbf{j}}|i\rangle .
\end{aligned}
$$

Commuting the Hamiltonian $\bar{H}-\bar{H}_{\mathbf{m} \mathbf{j}}$ (which does not contain operators on site $\mathbf{j}$ ) with the operator $D_{\mathbf{j}, \mathbf{e}^{i}}$ and $P_{\mathbf{j}}$ gives 


$$
\begin{aligned}
P_{\mathbf{j}} D_{\mathbf{j}, \mathbf{e}^{f}}^{\dagger}\left|L_{3}\right\rangle\left\langle L_{3}\left|\bar{H} D_{\mathbf{j}, \mathbf{e}^{i}} P_{\mathbf{j}}\right| i\right\rangle= & P_{\mathbf{j}} D_{\mathbf{j}, \mathbf{e}^{f}}^{\dagger}\left|L_{3}\right\rangle\left\langle L_{3}\right| \\
& \times D_{\mathbf{j}, \mathbf{e}^{i}} P_{\mathbf{j}}\left(\bar{H}-\bar{H}_{\mathbf{m} \mathbf{j}}\right)|i\rangle .
\end{aligned}
$$

Since $\bar{H}|i\rangle=H|i\rangle=E_{i}|i\rangle=0$ to set the energy to zero, we are left with the following expression: $P_{\mathbf{j}} D_{\mathbf{j}, \mathbf{e}^{f}}^{\dagger}\left|L_{3}\right\rangle\left\langle L_{3}\left|D_{\mathbf{j}, \mathbf{e}^{i}} P_{\mathbf{j}} \bar{H}_{\mathbf{m} \mathbf{j}}\right| i\right\rangle$. However, because of $P_{\mathbf{j}}$, the $U$ and the $U_{c}$ terms will never contribute, and $\left.P_{\mathbf{j}} D_{\mathbf{j}, \mathbf{e}^{f}}^{\dagger}\left|L_{3}\right\rangle\left\langle L_{3}\right| D_{\mathbf{j}, \mathbf{e}^{i}} P_{\mathbf{j}} d_{\mathbf{l} \sigma}^{\dagger} d_{\mathbf{j} \sigma}\right)|i\rangle=0$ for any $\mathbf{l}=\mathbf{m}, \mathbf{m}^{\prime}$. Thus, we obtain

$$
\begin{aligned}
P_{\mathbf{j}} D_{\mathbf{j}, \mathbf{e}^{f}}^{\dagger} & \left|L_{3}\right\rangle\left\langle L_{3}\left|\bar{H} D_{\mathbf{j}, \mathbf{e}^{i}} P_{\mathbf{j}}\right| i\right\rangle \\
= & -P_{\mathbf{j}} D_{\mathbf{j}, \mathbf{e}^{f}}^{\dagger}\left|L_{3}\right\rangle\left\langle L_{3}\right| D_{\mathbf{j}, \mathbf{e}^{i}} \\
& \times\left[-t \sum_{\mathbf{m}(\mathbf{j}), \sigma}\left(d_{\mathbf{j} \sigma}^{\dagger} d_{\mathbf{m} \sigma}\right)-t^{\prime} \sum_{\mathbf{m}^{\prime}(\mathbf{j}), \sigma}\left(d_{\mathbf{j} \sigma}^{\dagger} d_{\mathbf{m} \sigma}\right)\right] P_{\mathbf{j}}|i\rangle .
\end{aligned}
$$

Note the asymmetry in the above expression, i.e., the lack of the Hermitian conjugate terms $\propto d_{j \sigma}^{\dagger} d_{m \sigma}$-this asymmetry expresses the fact that in RIXS we are only sensitive to sites on which the $3 d$ holes reside.

Introducing so-called local matrix elements of RIXS, we obtain

$$
\frac{1}{i \Gamma} P_{\mathbf{j}} D_{\mathbf{j}, \mathbf{e}^{f}}^{\dagger}\left|L_{3}\right\rangle\left\langle L_{3}\right| D_{\mathbf{j}, \mathbf{e}^{i}} P_{\mathbf{j}} \equiv W_{\mathbf{e}} P_{\mathbf{j}} n_{j} P_{\mathbf{j}}+\tilde{W}_{\mathbf{e}} P_{\mathbf{j}} S_{\mathbf{j}}^{z} P_{\mathbf{j}}
$$

where the local RIXS form factors follow from, e.g., Ref. [32] [cf. Eq. (2) and Fig. 1 in Ref. [32]]:

$$
\begin{aligned}
& W_{\pi-\sigma}=-\imath 2\left(\mathbf{e}_{y}^{i} \mathbf{e}_{x}^{f}-\mathbf{e}_{x}^{i} \mathbf{e}_{y}^{f}\right) /(3 \Gamma), \\
& W_{\pi-\pi}=-2\left(\mathbf{e}_{x}^{i} \mathbf{e}_{x}^{f}+\mathbf{e}_{y}^{i} \mathbf{e}_{y}^{f}\right) /(3 \Gamma) .
\end{aligned}
$$

Let $P_{\mathbf{j}} n_{\mathbf{j}} P_{\mathbf{j}}=\tilde{n}_{\mathbf{j}}$ [where $\tilde{n}_{\mathbf{j}}=\sum_{\sigma} \tilde{n}_{\mathbf{j} \sigma}=\sum_{\sigma} \tilde{d}_{\mathbf{j} \sigma}^{\dagger} \tilde{d}_{\mathbf{j} \sigma}$ and $\left.\tilde{d}_{\mathbf{j} \sigma}^{\dagger}=d_{\mathbf{j} \sigma}^{\dagger}\left(1-n_{\mathbf{j},-\sigma}\right)\right]$ and $P_{\mathbf{j}} S_{\mathbf{j}}^{z} P_{\mathbf{j}}=S_{\mathbf{j}}^{z}$. Combining the above equations, we finally arrive at the expression for the RIXS operators in the UCL approximation,

$$
\begin{aligned}
O_{\mathbf{j}, \mathbf{e}}^{(1)}= & W_{\mathbf{e}} \tilde{n}_{\mathbf{j}}+\tilde{W}_{\mathbf{e}} S_{\mathbf{j}}^{z}, \\
O_{\mathbf{j}, \mathbf{e}}^{(2)}= & \frac{t}{i \Gamma} W_{\mathbf{e}} \sum_{\mathbf{m}(\mathbf{j})} \tilde{d}_{\mathbf{j} \sigma}^{\dagger} d_{\mathbf{m} \sigma}+\frac{t}{i \Gamma} \tilde{W}_{\mathbf{e}} S_{\mathbf{j}}^{z} \sum_{\mathbf{m}(\mathbf{j})} d_{\mathbf{j} \sigma}^{\dagger} d_{\mathbf{m} \sigma} \\
& +\frac{t^{\prime}}{i \Gamma} W_{\mathbf{e}} \sum_{\mathbf{m}^{\prime}(\mathbf{j})} \tilde{d}_{\mathbf{j} \sigma}^{\dagger} d_{\mathbf{m} \sigma}+\frac{t^{\prime}}{i \Gamma} \tilde{W}_{\mathbf{e}} S_{\mathbf{j}}^{z} \sum_{\mathbf{m}^{\prime}(\mathbf{j})} d_{\mathbf{j} \sigma}^{\dagger} d_{\mathbf{m} \sigma} .
\end{aligned}
$$

Substituting the above expressions into Eq. (1) and performing Fourier transformations, we obtain Eqs. (6) and (9). As the first- (second-)order UCL terms have real (imaginary) contributions, the interference terms vanish and the full RIXS cross section consists of separate first- and second-order UCL terms. Note that if only these first two terms are considered in the UCL approximation, then the RIXS spectrum does not depend on the size of the core-hole potential $U_{c}$ (the latter will appear only in higher-order corrections in the UCL approximation).

\section{APPENDIX B: UCL APPROXIMATION FOR THE $t-J$ MODEL}

For completeness, we have evaluated the UCL expansion of the RIXS cross section for the $t-J$ model- the strong coupling expansion of the Hubbard model, valid for the low-energy physics well below the energy scale $U=8 t$. For the qualitative discussions here, we can safely neglect $t^{\prime}$ and the three-site terms in this expansion. Following similar steps as described in the previous section for the Hubbard model, we obtain, for the $\pi-\sigma$ channel,

$$
\begin{aligned}
I_{\pi-\sigma}^{\mathrm{UCL}}(\mathbf{q}, \omega)= & \left|W_{\pi-\sigma}\right|^{2}\left\{\sum_{f}\left|\left\langle f\left|S_{\mathbf{q}}^{z}\right| i\right\rangle\right|^{2} \delta\left(\omega+E_{i}-E_{f}\right)+\frac{z^{2} J^{2}}{N^{2} \Gamma^{2}} \sum_{f}\left|\left\langle f\left|\sum_{\mathbf{k}, \mathbf{k}^{\prime}} \gamma_{\mathbf{k}^{\prime}+\mathbf{k}-\mathbf{q}} S_{\mathbf{k}^{\prime}}^{z} \mathbf{S}_{\mathbf{k}} \mathbf{S}_{-\mathbf{k}^{\prime}-\mathbf{k}+\mathbf{q}}\right| i\right\rangle\right|^{2} \delta\left(\omega+E_{i}-E_{f}\right)\right. \\
& \left.+\frac{z^{2} t^{2}}{N^{2} \Gamma^{2}} \sum_{f}\left|\left\langle f\left|\sum_{\mathbf{k}, \mathbf{k}^{\prime}} S_{\mathbf{k}^{\prime}}^{z} \gamma_{-\mathbf{k}^{\prime}+\mathbf{k}+\mathbf{q}} \tilde{d}_{\mathbf{k}, \sigma}^{\dagger} \tilde{d}_{-\mathbf{k}^{\prime}+\mathbf{k}+\mathbf{q}, \sigma}\right| i\right\rangle\right|^{2} \delta\left(\omega+E_{i}-E_{f}\right)\right\},
\end{aligned}
$$

and for the $\pi-\pi$ channel,

$$
\begin{aligned}
I_{\pi-\pi}^{\mathrm{UCL} L_{t-J}}(\mathbf{q}, \omega)= & \left|W_{\pi-\pi}\right|^{2}\left\{\sum_{f}\left|\left\langle f\left|\tilde{n}_{\mathbf{q}}\right| i\right\rangle\right|^{2} \delta\left(\omega+E_{i}-E_{f}\right)+\frac{z^{2} J^{2}}{N \Gamma^{2}} \sum_{f}\left|\left\langle f\left|\sum_{\mathbf{k}} \gamma_{\mathbf{q}-\mathbf{k}} \mathbf{S}_{\mathbf{k}} \mathbf{S}_{-\mathbf{k}+\mathbf{q}}\right| i\right\rangle\right|^{2} \delta\left(\omega+E_{i}-E_{f}\right)\right. \\
& \left.+\frac{z^{2} t^{2}}{N \Gamma^{2}} \sum_{f}\left|\left\langle f\left|\sum_{\mathbf{k}} \gamma_{\mathbf{q}+\mathbf{k}} \tilde{d}_{\mathbf{k}, \sigma}^{\dagger} \tilde{d}_{\mathbf{q}+\mathbf{k}, \sigma}\right| i\right\rangle\right|^{2} \delta\left(\omega+E_{i}-E_{f}\right)\right\} .
\end{aligned}
$$


Here, $J=4 t^{2} / U$, and the spin operators are defined in a standard way as

$$
\begin{aligned}
\mathbf{S}_{\mathbf{k}} \mathbf{S}_{-\mathbf{q}-\mathbf{k}}= & \frac{1}{2 N} \sum_{\mathbf{q}_{1}, \mathbf{q}_{2}, \sigma} \tilde{d}_{\mathbf{q}_{1}, \sigma}^{\dagger} \tilde{\sigma}_{\mathbf{q}_{1}+\mathbf{q}, \bar{\sigma}} \tilde{d}_{\mathbf{q}_{2}, \bar{\sigma}}^{\dagger} \tilde{d}_{\mathbf{q}_{2}-\mathbf{q}-\mathbf{k}, \sigma} \\
& +\frac{1}{4 L} \sum_{\mathbf{q}_{1}, \mathbf{q}_{2}}\left(\tilde{d}_{\mathbf{q}_{1}, \uparrow}^{\dagger} \tilde{d}_{\mathbf{q}_{1}+\mathbf{k}, \uparrow}-\tilde{d}_{\mathbf{q}_{1}, \downarrow}^{\dagger} \tilde{d}_{\mathbf{q}_{1}+\mathbf{k}, \downarrow}\right) \\
& \times\left(\tilde{d}_{\mathbf{q}_{2}, \uparrow}^{\dagger} \tilde{d}_{\mathbf{q}_{2}-\mathbf{q}-\mathbf{k}, \uparrow}-\tilde{d}_{\mathbf{q}_{2}, \downarrow}^{\dagger} \tilde{d}_{\mathbf{q}_{2}-\mathbf{q}-\mathbf{k}, \downarrow}\right) .
\end{aligned}
$$

Note that all the operators $\tilde{d}_{\mathbf{i}, \sigma}$ and $\tilde{d}_{\mathbf{i}, \sigma}^{\dagger}$ are defined in the constrained Hilbert space without double occupancies.

These equations show that in the second order of the UCL expansion, we obtain two sets of terms. The first terms contain only the spin operators and correspond to the "two-spin"

$$
\begin{aligned}
S_{2}(\mathbf{q}, \omega)= & \frac{z^{2} J^{2}}{N \Gamma^{2}} \sum_{f}\left|\left\langle f\left|\sum_{\mathbf{k}} \gamma_{\mathbf{q}-\mathbf{k}} \mathbf{S}_{\mathbf{k}} \mathbf{S}_{\mathbf{k}-\mathbf{q}}\right| i\right\rangle\right|^{2} \\
& \times \delta\left(\omega+E_{i}-E_{f}\right),
\end{aligned}
$$

and "three-spin" Green's functions

$$
\begin{aligned}
S_{3}(\mathbf{q}, \omega)= & \frac{z^{2} J^{2}}{L^{2} \Gamma^{2}} \sum_{f} \mid\langle f| \sum_{\mathbf{k}, \mathbf{k}^{\prime}} \gamma_{\mathbf{k}^{\prime}+\mathbf{k}-\mathbf{q}} S_{\mathbf{k}^{\prime}}^{z} \\
& \times\left.\mathbf{S}_{\mathbf{k}} \mathbf{S}_{-\mathbf{k}^{\prime}-\mathbf{k}+\mathbf{q}}|i\rangle\right|^{2} \delta\left(\omega+E_{i}-E_{f}\right) .
\end{aligned}
$$

The second terms always involve charge excitations below the gap. Since these terms do not contribute at half filling (because of the constrained Hilbert space without double occupancies), it is expected that at relatively low doping levels, the first terms should be dominant (even though their amplitude scales with $J / \Gamma$ and not with $t / \Gamma$ ). Hence, we compare the spectra of these first two terms, $S_{2}(\mathbf{q}, \omega)$ and $S_{3}(\mathbf{q}, \omega)$, in the second order of the UCL expansion with the spectra of $S^{\prime}(\mathbf{q}, \omega)$ and $\tilde{N}^{\prime}(\mathbf{q}, \omega)$ (cf. Fig. 6) from the Hubbard model. We see that at half filling, $S^{\prime}(\mathbf{q}, \omega)$ can be approximated relatively well by the three-spin excitations probed by $S_{3}(\mathbf{q}, \omega)$ and that $\tilde{N}^{\prime}(\mathbf{q}, \omega)$ can be approximated relatively well by the two-spin or bimagnon excitations probed by $S_{2}(\mathbf{q}, \omega)$. Most of the discrepancies between these two spectra can be found in the high-energy regime and are therefore attributed to the failure of the $t-J$ model expansion at higher energies. The electron- and hole-doped cases show much less pronounced agreement, where the projected spin and charge excitations $S^{\prime}(\mathbf{q}, \omega)$ and $\tilde{N}^{\prime}(\mathbf{q}, \omega)$ also probe the low-energy charge excitations below the gap, which can give a relatively large contribution in the spectrum.

The prediction that RIXS can probe the two-spin and the three-spin excitations at half filling has already been
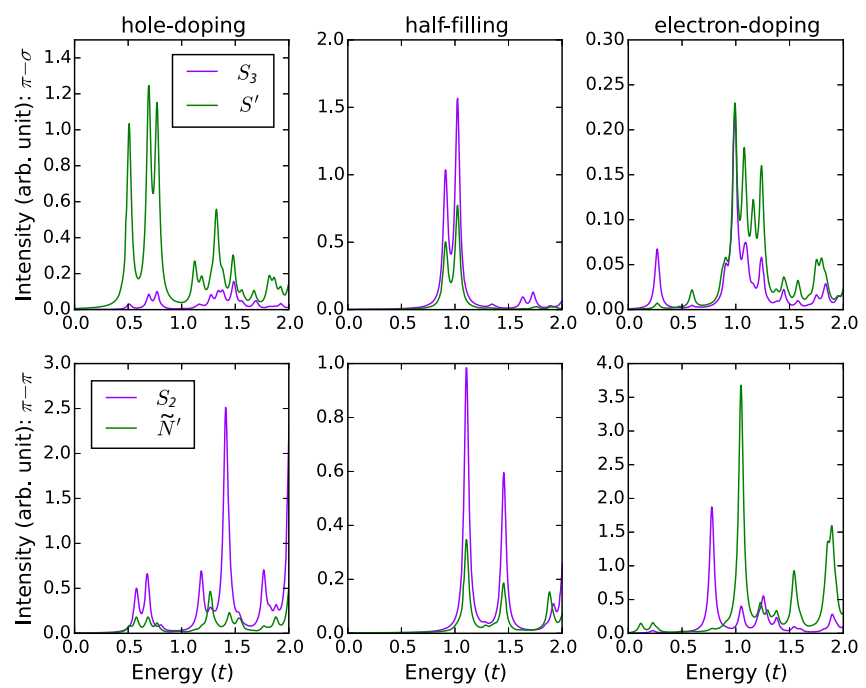

FIG. 6. Comparison between $S^{\prime}$ and three-spin Green's functions $S_{3}$ and between $\tilde{N}^{\prime}$ and two-spin Green's functions $S_{2}$ calculated using exact diagonalization and following Eqs. (8) and (11) from the main text of the paper and Eqs. (B4) and (B5). The top (bottom) panels show spectra for the $\pi-\sigma(\pi-\pi)$ polarization setups (see main text of the paper for further details). Left, middle, and right panels show RIXS spectra calculated for $n=0.83, n=1$, and $n=1.17$ electron fillings, respectively. The spectra are summed over all momenta along the nodal direction and along the $k_{y}=0$ direction that are accessible in RIXS and on a 12-site cluster used in the exact diagonalization calculations and weighted with the RIXS form factors, i.e., $S^{\prime}(\omega)=\sum_{\mathbf{q}}\left|W_{\pi-\sigma}\right|^{2} S^{\prime}(\mathbf{q}, \omega), \tilde{N}^{\prime}(\omega)=\sum_{\mathbf{q}}\left|W_{\pi-\pi}\right|^{2} \tilde{N}^{\prime}(\mathbf{q}, \omega)$, $S_{2}(\omega)=\sum_{\mathbf{q}}\left|W_{\pi-\pi}\right|^{2} S_{2}(\mathbf{q}, \omega)$, and $S_{3}(\omega)=\sum_{\mathbf{q}}\left|W_{\pi-\sigma}\right|^{2} S_{3}(\mathbf{q}, \omega)$. The intensity scale is different on each panel (it is chosen in such a way that each of the six spectra can be quite visible), and the elastic response has been removed.

put forward in Refs. [34-37,54,55] in the case of the Heisenberg model, consistent with our UCL expansions for the Hubbard and $t-J$ models. Note that usually the Green's functions containing the two-spin and the threespin operators are referred to as probing the "two-magnon" and the "three-magnon" spectrum, though this terminology may be used loosely in this context. Finally, the fact that the charge dynamical structure factor for the half-filled Hubbard model probes the two-magnon spectrum has also been discussed in the context of nonresonant Raman scattering (cf. Refs. [[23,56,57]]).

\section{APPENDIX C: X-RAY ABSORPTION SPECTROSCOPY}

Although the intent of this manuscript is not to directly compare the calculated spectra with the experimental results, we show the calculated XAS for completeness. In what follows, we also discuss the nonlocal character of the wavefunction of the RIXS intermediate states by comparing the XAS spectrum calculated on a single site 
(a) single-site
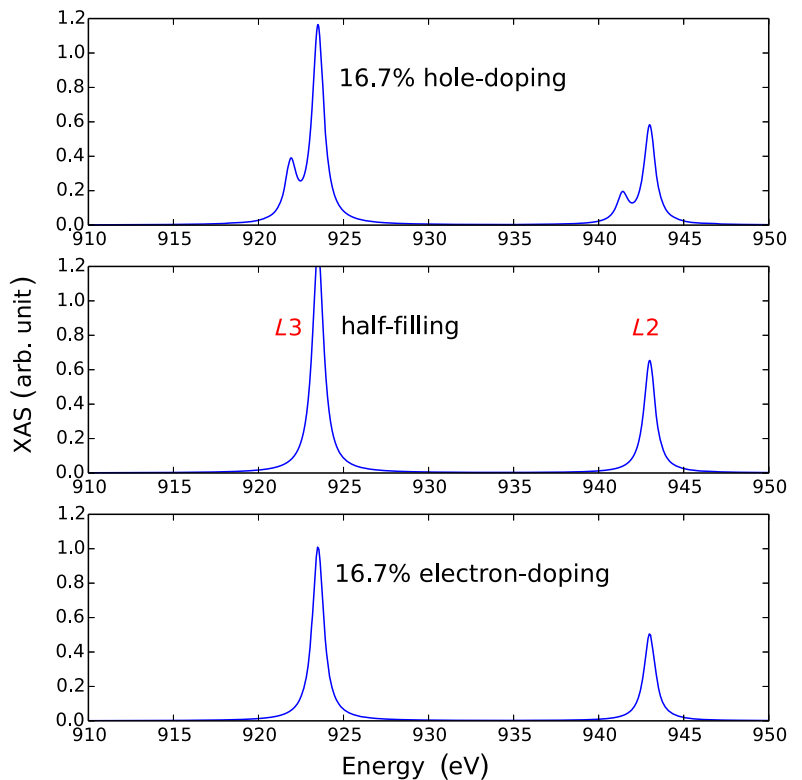

(b) 12-site cluster
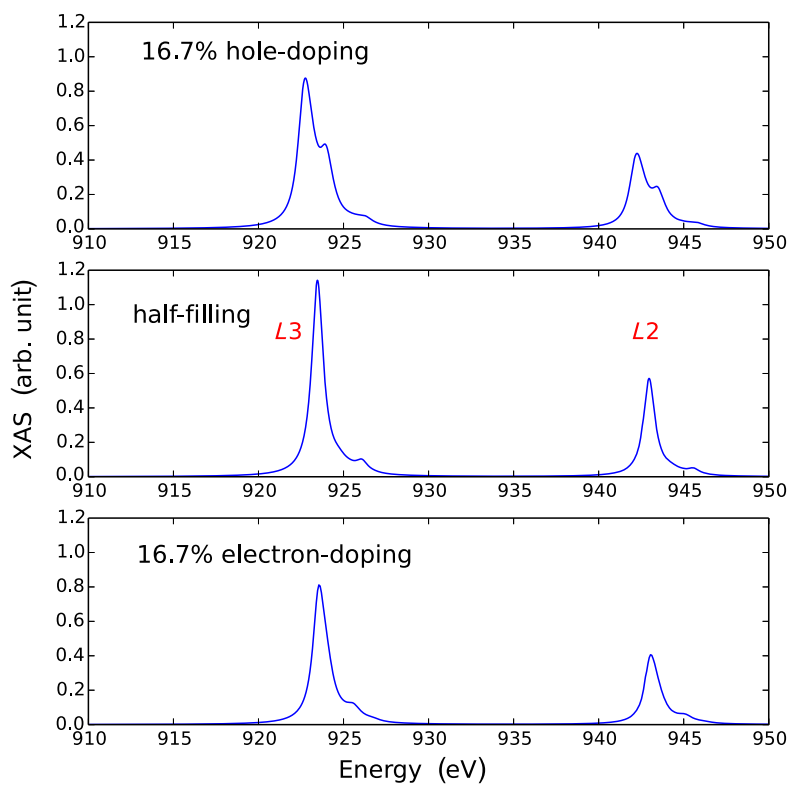

FIG. 7. Comparison between the $\mathrm{Cu} L$-edge XAS spectrum calculated using exact diagonalization on a single site (top three panels) and on a 12-site cluster with both hopping $t$ and $t^{\prime}$ taken into account in the Hubbard model (bottom three panels) for holedoped, half-filling, and electron-doped cases (see text for more details). In this paper, RIXS is always calculated for the resonance corresponding to the XAS transition from the $2 p^{0} 3 d^{* 1}$ to the $2 p^{1} 3 d^{* 0}$ state and the $L_{3}$ edge $(923 \mathrm{eV}$ peak in the above spectra).

with the XAS calculated on a 12-site cluster (i.e., the same cluster as used for RIXS calculation) (see Fig. 7). It can be easily verified that the single-site calculations yield a substantially more distinct XAS spectrum than the cluster calculations: The XAS spectrum not only consists of the two main XAS peaks (four in the hole-doped case), which are visible in both calculations and which correspond to the $L_{2}$ and the $L_{3}$ edges, but it also consists of the much smaller shoulder peaks, which are only visible in the cluster calculations. These small shoulder peaks, which lie at energy ca. $925 \mathrm{eV}(945-946 \mathrm{eV})$ for the $L_{3}\left(L_{2}\right)$ edge and remain there for all doping levels, correspond to the XAS final state with the delocalized $3 d^{* 0}$ configuration, and cannot be obtained in the single-site calculations. This shows that the XAS final states have nonlocal character.

The above result has an important consequence for RIXS: Since the XAS final states have nonlocal character, it is expected that the intermediate states of RIXS cannot be properly described in the single-site approximation. Since the latter essentially corresponds to the fast collision approximation, it is rather natural to expect that such approximation may fail in describing the RIXS cross section (which indeed occurs-see the main text of the paper).

We note that there is also an additional peak in the holedoped XAS spectrum, visible both in the single-site and in the cluster calculations at ca. $922 \mathrm{eV}(942 \mathrm{eV})$ for the $L_{3}$ $\left(L_{2}\right)$ edge. This peak corresponds to the XAS performed on the hole-doped sites, i.e., to the transitions from the $2 p^{0} 3 d^{* 2}$ to the $2 p^{1} 3 d^{* 1}$ configuration: Since $U_{c}<U$ in our calculations, this transition costs less energy than the "main" XAS transition, which corresponds to the transition from the $2 p^{0} 3 d^{* 1}$ to the $2 p^{1} 3 d^{* 0}$ (923 eV at the $L_{3}$ and $943 \mathrm{eV}$ at the $L_{2}$ edge). Because of the strongly nonlocal character of the initial $2 p^{0} 3 d^{* 2}$ configuration, the XAS performed on these "doubly occupied" sites has a larger amplitude than the XAS transition performed on the singly occupied sites. The latter is a peculiarity of the single-band Hubbard model and will not be visible in the more experimentally relevant charge transfer model or in the experimental XAS spectra of the cuprates. Note, however, that since in our calculations we tune to the resonance corresponding to the $2 p^{0} 3 d^{* 1} \rightarrow 2 p^{1} 3 d^{* 0}$ XAS transition, the details of the $2 p^{0} 3 d^{* 2}$ to the $2 p^{1} 3 d^{* 1}$ XAS transition are not that important (at least in the first approximation). Thus, the partial disagreement between our calculated XAS and the experimentally observed XAS on the cuprates does not invalidate the application of the RIXS numerical results to the understanding of the RIXS.

\section{APPENDIX D: CORE-HOLE POTENTIAL DEPENDENCE}

In what follows, we show that the main conclusions of the paper hold independently of the value of the core-hole potential $U_{c}$-provided that this value is chosen within some realistic range. Let us first state that the assumed value of $U_{c} \sim 4 t$ is a reasonable estimate and has been utilized in the previous studies [24,58]. On the other hand, another study assumed a much larger $U_{c} \sim 15 t$ [59], and a recent simulation of the RIXS experiment used $U_{c}=6 t$ 
[60] to fit with experiments. One should mention that estimating $U_{c}$ from the first-principles calculations is rather difficult: The core-hole potential $U_{c}$ is a monopole term; as a result, the strong screening effect cannot be neglected, and simply calculating the Coulomb interaction in the Wannier basis (with the Wannier orbitals obtained from, e.g., density functional theory calculations) usually fails to provide a reasonable value of $U_{c}$ [61]. Moreover, in the single-band model, $U_{c}$ cannot be directly connected with $U_{c}$ of the three-band model, as the effective orbital $d$ in the single-band model is the linear combination of the ligand oxygen and not directly related to the $\mathrm{Cu} d$ orbital. In the end, one can treat $U_{c}$ as a free parameter and fit with experiments (fitting with experiment is beyond the scope of our study).

Altogether, the above analysis suggests that $U_{c} \sim 4-$ $8 t$ may be a good estimate for $3 d$ transition metal oxides, such as cuprates. While in the main text we calculated the RIXS spectra for $U_{c}=4 t$, here we investigate how the RIXS spectra change when a larger (but still realistic) $U_{c}=8 t$. The calculated RIXS spectra using the exact diagonalization method for the Hubbard model at half filling (for details, see main text of the paper) are shown in Fig. 8 (for didactic reasons, spectra for a rather unrealistic value of $U_{c}=2 t$ are also presented). Note that since the RIXS spectra are plotted using the same
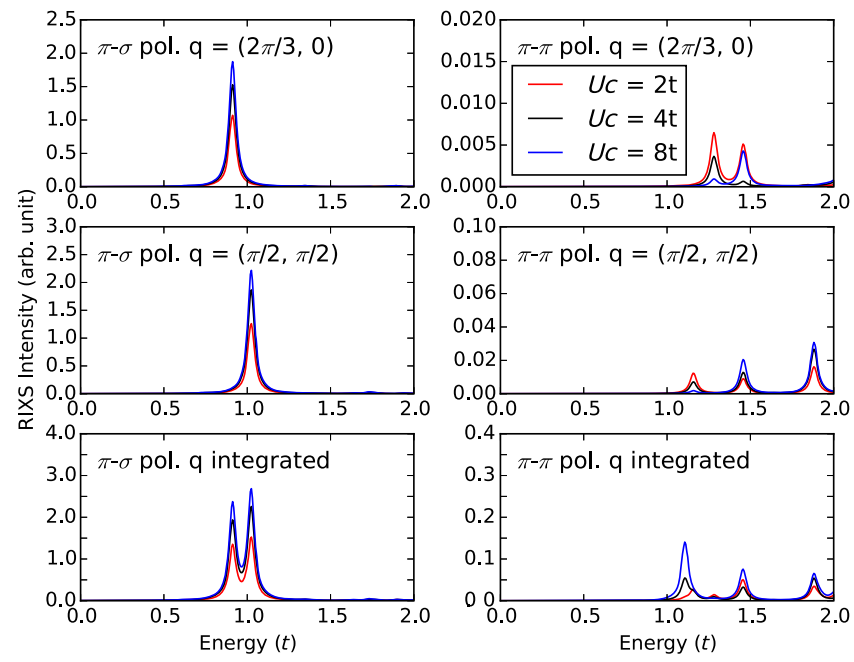

FIG. 8. Exact RIXS cross section calculated using exact diagonalization for $n=1$ (half filling) and for three different values of the core-hole potential: $U_{c}=2 t, 4 t$, and $8 t$ [the model, its parameters (except for $U_{c}$ ), and the exact diagonalization method are the same as in the main text]. The left (right) panels show the RIXS spectra for the $\pi-\sigma(\pi-\pi)$ relative polarization. The top, middle, and bottom panels show spectra calculated at $\mathbf{q}=(\pi / 2, \pi / 2), \mathbf{q}=(2 \pi / 3,0)$, and integrated over all momenta along the nodal direction and along the $q_{y}=0$ direction accessible on a 12-site cluster ("q integrated"). Elastic response is excluded, and each RIXS spectrum is calculated at the incident energy corresponding to the main (" $L_{3}$ ") XAS peak at half filling. scale as in those presented in the main text, one can easily compare the spectra for different $U_{c}$. We see that increasing $U_{c}$ indeed changes the total spectral weight carried by the magnetic peak in the cross-polarized channel and leads to a transfer of spectral weight between the peaks detected in the parallel-polarized channel. A more detailed investigation shows that RIXS spectra for $U_{c}=8 t$ are in better agreement with the approximate spectra obtained in the UCL approximation presented in the main text. Nevertheless, even in this case, this agreement between the approximate RIXS spectra and the exact RIXS spectra is not perfect: This mismatch is especially noticeable for the parallel-polarized case but also visible in the cross-polarized case. Finally, note also that the above comparison only takes into account the half-filled case, i.e., the case when the approximate and the exact RIXS spectra have the best agreement based on the $U_{c}=4 t$ results.

Intuitively, the fact that the agreement between approximate and exact RIXS becomes better when $U_{c}$ increases can be attributed to the decreasing $2 p^{1} 3 d^{* 1}$ feature in the RIXS intermediate state: The larger the probability of having solely sites with the $2 p^{1} 3 d^{* 0}$ configuration, the closer one is to the "single-ion" approximation-for which the approximate RIXS cross section becomes exact.

\section{APPENDIX E: VISUALIZATION OF THE FINAL-STATE CONFIGURATIONS}

Figure 9 shows the dominant $\mathrm{Cu} L$-edge RIXS process in the cross-polarized channel in hole language. The hole in the $3 d^{*}$ orbital on a particular site $\mathbf{j}$ in the initial state of RIXS is transferred via the dipole operator $D$ into the $2 p$ orbital on the same site $\mathbf{j}$ with a nonuniquely defined spin in the intermediate state of RIXS due to spin-orbit coupling in the core. This is transferred back via the dipole operator $D^{\dagger}$ to the $d$ orbital on the same site $\mathbf{j}$ with a spin flip in the final state compared to the initial state of the RIXS process. While we demonstrate this process on a single site $\mathbf{j}$ in real space, in reality a coherent superposition of such excitations is created with phase factors $e^{\mathbf{1 q j}}$; this leads to a welldefined, single spin flip with momentum $\mathbf{q}$ in the final state of RIXS-i.e., RIXS is sensitive to the spin dynamical structure factor $S(\mathbf{q}, \omega)$.

Figure 10 shows the dominant $\mathrm{Cu} L$-edge RIXS process in the parallel-polarized channel in hole language. The hole in the $3 d^{*}$ orbital on a particular site $\mathbf{j}$ in the initial state of RIXS is transferred via the dipole operator $D$ into the $2 p$ orbital on the same site $\mathbf{j}$ with a well-defined spin in the intermediate state. In the intermediate state, a "shakeup" happens, which creates a two-spin and/ or charge excitation in the final state. While this process is shown on a single site $\mathbf{j}$ (and its neighbors) in real space, in reality a coherent superposition of such excitations is created with a phase factor $e^{\mathbf{1 q j}}$; leading to a two-spin or charge excitation 


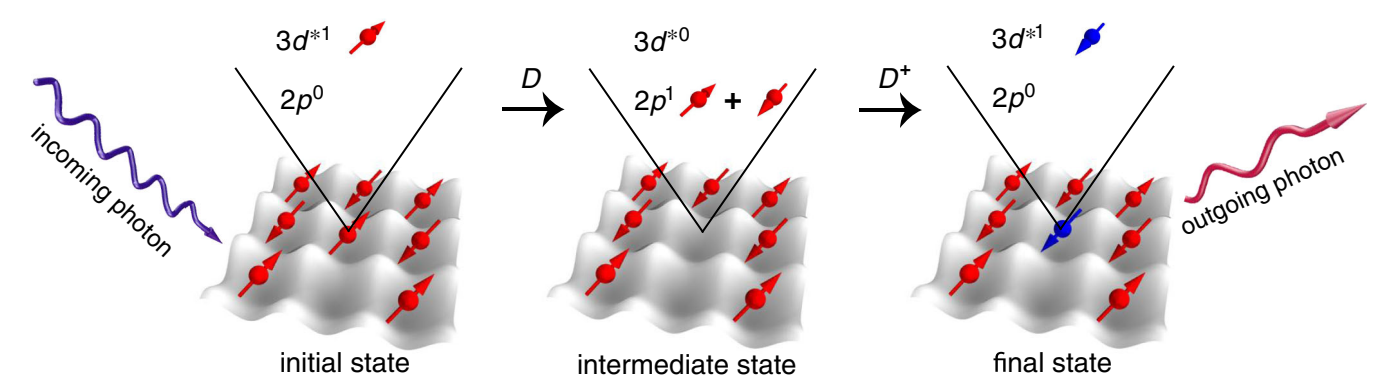

FIG. 9. A cartoon picture of the dominant $\mathrm{Cu} L$-edge RIXS process in the cross-polarized "channel."
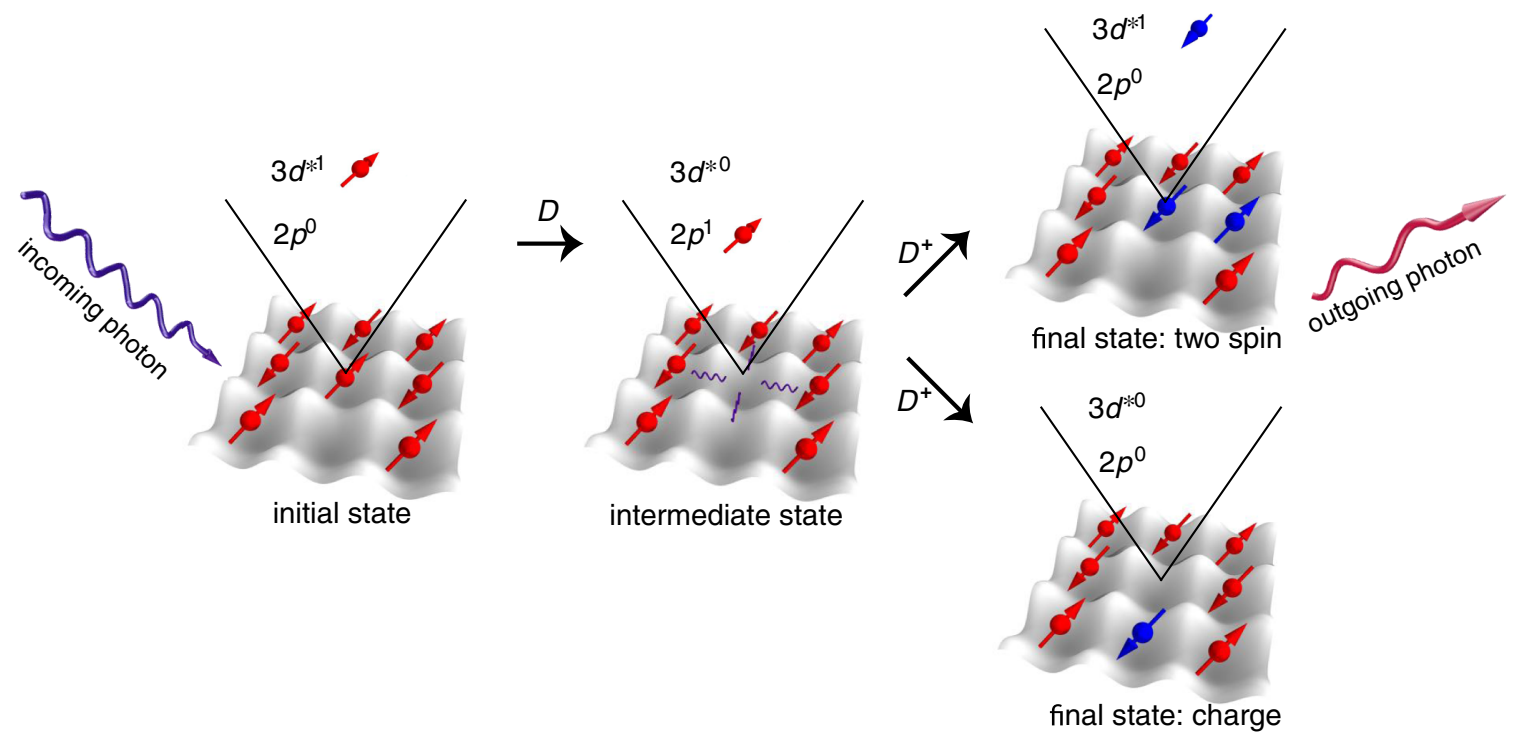

FIG. 10. A cartoon picture of the dominant $\mathrm{Cu} L$-edge RIXS process in the parallel-polarized channel.

created with momentum $\mathbf{q}$ in the final state; i.e., RIXS has some sensitivity to $\tilde{N}^{\prime}(\mathbf{q}, \omega)$, which unfortunately has no simple analog in the standard two-particle charge response function.

[1] L. J. P. Ament, M. van Veenendaal, T. P. Devereaux, J. P. Hill, and J. van den Brink, Resonant Inelastic X-Ray Scattering Studies of Elementary Excitations, Rev. Mod. Phys. 83, 705 (2011).

[2] A. Kotani and S. Shin, Resonant Inelastic X-ray Scattering Spectra for Electrons in Solids, Rev. Mod. Phys. 73, 203 (2001).

[3] J. Schlappa, T. Schmitt, F. Vernay, V. N. Strocov, V. Ilakovac, B. Thielemann, H. M. Rønnow, S. Vanishri, A. Piazzalunga, X. Wang, L. Braicovich, G. Ghiringhelli, C. Marin, J. Mesot, B. Delley, and L. Patthey, Collective Magnetic Excitations in the Spin Ladder $\mathrm{Sr}_{14} \mathrm{Cu}_{24} \mathbf{O}_{41}$ Measured Using High-Resolution Resonant Inelastic XRay Scattering, Phys. Rev. Lett. 103, 047401 (2009).

[4] L. Braicovich, L. J. P. Ament, V. Bisogni, F. Forte, C. Aruta, G. Balestrino, N. B. Brookes, G. M. De Luca, P. G. Medaglia, F. Miletto Granozio et al., Dispersion of
Magnetic Excitations in the Cuprate $\mathrm{La}_{2} \mathrm{CuO}_{4}$ and $\mathrm{CaCuO}_{2}$ Compounds Measured Using Resonant X-Ray Scattering, Phys. Rev. Lett. 102, 167401 (2009).

[5] M. Guarise, B. Dalla Piazza, M. Moretti Sala, G. Ghiringhelli, L. Braicovich, H. Berger, J. N. Hancock, D. van der Marel, T. Schmitt, V. N. Strocov et al., Measurement of Magnetic Excitations in the Two-Dimensional Antiferromagnetic $\mathrm{Sr}_{2} \mathrm{CuO}_{2} \mathrm{Cl}_{2}$ Insulator Using Resonant X-Ray Scattering: Evidence for Extended Interactions, Phys. Rev. Lett. 105, 157006 (2010).

[6] L. Braicovich, J. van den Brink, V. Bisogni, M. Moretti Sala, L. J. P. Ament, N. B. Brookes, G. M. De Luca, M. Salluzzo, T. Schmitt, V. N. Strocov, and G. Ghiringhelli, Magnetic Excitations and Phase Separation in the Underdoped $\mathrm{La}_{2-x} \mathrm{Sr}_{x} \mathrm{CuO}_{4}$ Superconductor Measured by Resonant Inelastic X-Ray Scattering, Phys. Rev. Lett. 104, 077002 (2010).

[7] J. Schlappa, K. Wohlfeld, K. J. Zhou, M. Mourigal, M. W. Haverkort, V. N. Strocov, L. Hozoi, C. Monney, S. Nishimoto, S. Singh et al., Spin-Orbital Separation in the Quasi-One-Dimensional Mott Insulator $\mathrm{Sr}_{2} \mathrm{CuO}_{3}$, Nature (London) 485, 82 (2012).

[8] M. Le Tacon, G. Ghiringhelli, J. Chaloupka, M. Moretti Sala, V. Hinkov, M. W. Haverkort, M. Minola, M. Bakr, K. J. Zhou, S. Blanco-Canosa et al., Intense Paramagnon 
Excitations in a Large Family of High-Temperature Superconductors, Nat. Phys. 7, 725 (2011).

[9] M. P. M. Dean, A. J. A. James, R. S. Springell, X. Liu, C. Monney, K. J. Zhou, R. M. Konik, J. S. Wen, Z. J. Xu, G. D. $\mathrm{Gu}$ et al., High-Energy Magnetic Excitations in the Cuprate Superconductor $\mathrm{Bi}_{2} \mathrm{Sr}_{2} \mathrm{CaCu}_{2} \mathrm{O}_{8+\delta}$ : Towards a Unified Description of Its Electronic and Magnetic Degrees of Freedom, Phys. Rev. Lett. 110, 147001 (2013).

[10] M. P. M. Dean, G. Dellea, R. S. Springell, F. Yakhou-Harris, K. Kummer, N. B. Brookes, X. Liu, Y.-J. Sun, J. Strle, T. Schmitt et al., Persistence of Magnetic Excitations in $\mathrm{La}_{2-x} \mathrm{Sr}_{x} \mathrm{CuO}_{4}$ from the Undoped Insulator to the Heavily Overdoped Non-superconducting Metal, Nat. Mater. 12, 1019 (2013).

[11] M. P. M. Dean, G. Dellea, M. Minola, S. B. Wilkins, R. M. Konik, G. D. Gu, M. Le Tacon, N. B. Brookes, F. Yakhou-Harris, K. Kummer et al., Magnetic Excitations in Stripe-Ordered $\mathrm{La}_{1.875} \mathrm{Ba}_{0.125} \mathrm{CuO}_{4}$ Studied Using Resonant Inelastic X-ray Scattering, Phys. Rev. B 88, 020403 (2013).

[12] M. Le Tacon, M. Minola, D. C. Peets, M. Moretti Sala, S. Blanco-Canosa, V. Hinkov, R. Liang, D. A. Bonn, W. N. Hardy, C. T. Lin et al., Dispersive Spin Excitations in Highly Overdoped Cuprates Revealed by Resonant Inelastic X-Ray Scattering, Phys. Rev. B 88, 020501 (2013).

[13] K. Ishii, M. Fujita, T. Sasaki, M. Minola, G. Dellea, C. Mazzoli, K. Kummer, G. Ghiringhelli, L. Braicovich, T. Tohyama et al., High-Energy Spin and Charge Excitations in Electron-Doped Copper Oxide Superconductors, Nat. Commun. 5, 3714 (2014).

[14] W. S. Lee, J. J. Lee, E. A. Nowadnick, W. Tabis, S. W. Huang, V. N. Strocov, E. M. Motoyama, G. Yu, B. Moritz, M. Greven et al., Asymmetry of Collective Excitations in Electron and Hole Doped Cuprate Superconductors, Nat. Phys. 10, 883 (2014).

[15] M. P. M. Dean, A. J. A. James, A. C. Walters, V. Bisogni, I. Jarrige, M. Hücker, E. Giannini, M. Fujita, J. Pelliciari, Y. B. Huang et al., Itinerant Effects and Enhanced Magnetic Interactions in Bi-based Multilayer Cuprates, Phys. Rev. B 90, 220506 (2014).

[16] M. Guarise, B. D. Piazza, H. Berger, E. Giannini, T. Schmitt, H. M. Rønnow, G. A. Sawatzky, J. van den Brink, D. Altenfeld, I. Eremin, and M. Grioni, Anisotropic Softening of Magnetic Excitations Along the Nodal Direction in Superconducting Cuprates, Nat. Commun. 5, 5760 (2014).

[17] M. Minola, G. Dellea, H. Gretarsson, Y. Y. Peng, Y. Lu, J. Porras, T. Loew, F. Yakhou, N. B. Brookes, Y. B. Huang et al., Collective Nature of Spin Excitations in Superconducting Cuprates Probed by Resonant Inelastic X-Ray Scattering, Phys. Rev. Lett. 114, 217003 (2015).

[18] S. Wakimoto, K. Ishii, H. Kimura, M. Fujita, G. Dellea, K. Kummer, L. Braicovich, G. Ghiringhelli, L. M. DebeerSchmitt, and G. E. Granroth, High-Energy Magnetic Excitations in Overdoped $\mathrm{La}_{2-x} \mathrm{Sr}_{x} \mathrm{CuO}_{4}$ Studied by Neutron and Resonant Inelastic X-Ray Scattering, Phys. Rev. B 91, 184513 (2015).

[19] P. Glatzel, U. Bergmann, J. Yano, H. Visser, J. H. Robblee, W. Gu, F. M. F. de Groot, G. Christou, V. L. Pecoraro, S. P. Cramer, and V. K. Yachandra, The Electronic Structure of Mn in Oxides, Coordination Complexes, and the OxygenEvolving Complex of Photosystem II Studied by Resonant
Inelastic X-Ray Scattering, J. Am. Chem. Soc. 126, 9946 (2004).

[20] S. G. Chiuzbăian, G. Ghiringhelli, C. Dallera, M. Grioni, P. Amann, X. Wang, L. Braicovich, and L. Patthey, Localized Electronic Excitations in $\mathrm{NiO}$ Studied with Resonant Inelastic X-Ray Scattering at the Ni M Threshold: Evidence of Spin Flip, Phys. Rev. Lett. 95, 197402 (2005).

[21] L. Braicovich, G. Ghiringhelli, L. H. Tjeng, V. Bisogni, C. Dallera, A. Piazzalunga, W. Reichelt, and N. B. Brookes, Neutral $3 d$ Excitations in Insulating $\mathrm{VO}_{2}$ as Seen with Resonant Inelastic X-Ray Scattering at the $V L_{3,2}$-Edges, Phys. Rev. B 76, 125105 (2007).

[22] D. J. Scalapino, A Common Thread: The Pairing Interaction for Unconventional Superconductors, Rev. Mod. Phys. 84, 1383 (2012).

[23] T. P. Devereaux and R. Hackl, Inelastic Light Scattering from Correlated Electrons, Rev. Mod. Phys. 79, 175 (2007).

[24] C. J. Jia, E. A. Nowadnick, K. Wohlfeld, Y. F. Kung, C.-C. Chen, S. Johnston, T. Tohyama, B. Moritz, and T. P. Devereaux, Persistent Spin Excitations in Doped Antiferromagnets Revealed by Resonant Inelastic Light Scattering, Nat. Commun. 5, 3314 (2014).

[25] C. J. Jia, C.-C. Chen, A. P. Sorini, B. Moritz, and T. P. Devereaux, Uncovering Selective Excitations Using the Resonant Profile of Indirect Inelastic X-Ray Scattering in Correlated Materials: Observing Two-Magnon Scattering and Relation to the Dynamical Structure Factor, New J. Phys. 14, 113038 (2012).

[26] Y. Wang, C. J. Jia, B. Moritz, and T. P. Devereaux, RealSpace Visualization of Remnant Mott Gap and Magnon Excitations, Phys. Rev. Lett. 112, 156402 (2014).

[27] L. J. P. Ament, G. Ghiringhelli, M. Moretti Sala, L. Braicovich, and J. van den Brink, Theoretical Demonstration of How the Dispersion of Magnetic Excitations in Cuprate Compounds Can Be Determined Using Resonant Inelastic X-Ray Scattering, Phys. Rev. Lett. 103, 117003 (2009).

[28] Jin Luo, G. T. Trammell, and J. P. Hannon, Scattering Operator for Elastic and Resonant Inelastic X-Ray Scattering, Phys. Rev. Lett. 71, 287 (1993).

[29] F. M. F. de Groot, P. Kuiper, and G. A. Sawatzky, Local Spin-Flip Spectral Distribution Obtained by Resonant X-Ray Raman Scattering, Phys. Rev. B 57, 14584 (1998).

[30] M. van Veenendaal, Polarization Dependence of $L$ - and M-Edge Resonant Inelastic X-Ray Scattering in TransitionMetal Compounds, Phys. Rev. Lett. 96, 117404 (2006).

[31] M. W. Haverkort, Theory of Resonant Inelastic X-Ray Scattering by Collective Magnetic Excitations, Phys. Rev. Lett. 105, 167404 (2010).

[32] P. Marra, K. Wohlfeld, and J. van den Brink, Unraveling Orbital Correlations with Magnetic Resonant Inelastic X-Ray Scattering, Phys. Rev. Lett. 109, 117401 (2012).

[33] L. J. P. Ament, F. Forte, and J. van den Brink, Ultrashort Lifetime Expansion for Indirect Resonant Inelastic X-Ray Scattering, Phys. Rev. B 75, 115118 (2007).

[34] V. Bisogni, L. Simonelli, L. J. P. Ament, F. Forte, M. Moretti Sala, M. Minola, S. Huotari, J. van den Brink, G. Ghiringhelli, N. B. Brookes, and L. Braicovich, Bimagnon Studies in Cuprates with Resonant Inelastic X-Ray Scattering at the $O \mathrm{~K}$-Edge. I. Assessment on $\mathrm{La}_{2} \mathrm{CuO}_{4}$ and 
Comparison with the Excitation at $\mathrm{Cu} \mathrm{L}_{3}$ and $\mathrm{Cu} \mathrm{K}$-Edges, Phys. Rev. B 85, 214527 (2012).

[35] V. Bisogni, M. Moretti Sala, A. Bendounan, N. B. Brookes, G. Ghiringhelli, and L. Braicovich, Bimagnon Studies in Cup Rates with Resonant Inelastic X-Ray Scattering at the $O$ K-Edge. II. Doping Effect in $\mathrm{La}_{2-x} \mathrm{Sr}_{x} \mathrm{CuO}_{4}$, Phys. Rev. B 85, 214528 (2012).

[36] Jun-I. Igarashi and T. Nagao, Magnetic Excitations in L-Edge Resonant Inelastic X-Ray Scattering from Cuprate Compounds, Phys. Rev. B 85, 064421 (2012).

[37] L. J. P. Ament and J. van den Brink, Strong Three-Magnon Scattering in Cuprates by Resonant X-Rays, arXiv: 1002.3773.

[38] D. Benjamin, I. Klich, and E. Demler, Single-Band Model of Resonant Inelastic X-Ray Scattering by Quasiparticles in High- $T_{c}$ Cuprate Superconductors, Phys. Rev. Lett. 112, 247002 (2014).

[39] F. C. Zhang and T. M. Rice, Effective Hamiltonian for the Superconducting Cu Oxides, Phys. Rev. B 37, 3759 (1988).

[40] R. B. Lehoucq, D. C. Sorensen, and C. Yang, ARPACK Users Guide: Solution of Large-Scale Eigenvalue Problems with Implicitly Restarted Arnoldi Methods (SIAM, Philadelphia, 1998).

[41] H. A. Van der Vorst, Bi-CGSTAB: A Fast and Smoothly Converging Variant of Bi-CG for the Solution of Nonsymmetric Linear Systems, SIAM J. Sci. Stat. Comput. 13, 631 (1992).

[42] E. Dagotto, Correlated Electrons in High-Temperature Superconductors, Rev. Mod. Phys. 66, 763 (1994).

[43] K. Wohlfeld, S. Nishimoto, M. W. Haverkort, and J. van den Brink, Microscopic Origin of Spin-Orbital Separation in $\mathrm{Sr}_{2} \mathrm{CuO}_{3}$, Phys. Rev. B 88, 195138 (2013).

[44] At this resonance, the intermediate state is an element of a subspace with no $3 d^{*}$ hole on the core-hole site.

[45] W. L. Yang, A. P. Sorini, C.-C. Chen, B. Moritz, W.-S. Lee, F. Vernay, P. Olalde-Velasco, J. D. Denlinger, B. Delley, J.-H. Chu et al., Evidence for Weak Electronic Correlations in Iron Pnictides, Phys. Rev. B 80, 014508 (2009).

[46] K.-J. Zhou, Y.-B. Huang, C. Monney, X. Dai, V. N. Strocov, N.-L. Wang, Z.-G. Chen, C. Zhang, P. Dai, L. Patthey, J. van den Brink, H. Ding, and T. Schmitt, Persistent High-Energy Spin Excitations in Iron-pnictide Superconductors, Nat. Commun. 4, 1470 (2013).

[47] L. A. Wray, J. Denlinger, S.-W. Huang, H. He, N. P. Butch, M. Brian Maple, Z. Hussain, and Y.-D. Chuang, Spectroscopic Determination of the Atomic f-Electron Symmetry Underlying Hidden Order in $\mathrm{URu}_{2} \mathrm{Si}_{2}$, Phys. Rev. Lett. 114, 236401 (2015).

[48] S. Moser, S. Fatale, P. Krüger, H. Berger, P. Bugnon, A. Magrez, H. Niwa, J. Miyawaki, Y. Harada, and M. Grioni, Electron-Phonon Coupling in the Bulk of Anatase $\mathrm{TiO}_{2}$ Measured by Resonant Inelastic X-Ray Spectroscopy, Phys. Rev. Lett. 115, 096404 (2015).
[49] M. Moretti Sala, V. Schnells, S. Boseggia, L. Simonelli, A. Al-Zein, J. G. Vale, L. Paolasini, E. C. Hunter, R. S. Perry, D. Prabhakaran et al., Evidence of Quantum Dimer Excitations in $\mathrm{Sr}_{3} \mathrm{Ir}_{2} \mathrm{O}_{7}$, Phys. Rev. B 92, 024405 (2015).

[50] E. Benckiser, L. Fels, G. Ghiringhelli, M. Moretti Sala, T. Schmitt, J. Schlappa, V. N. Strocov, N. Mufti, G. R. Blake, A. A. Nugroho et al., Orbital Superexchange and Crystal Field Simultaneously at Play in $\mathrm{YVO}_{3}$ : Resonant Inelastic $X$-Ray Scattering at the V L-Edge and the O K-Edge, Phys. Rev. B 88, 205115 (2013).

[51] Ph. Wernet, K. Kunnus, I. Josefsson, I. Rajkovic, W. Quevedo, M. Beye, S. Schreck, S. Grübel, M. Scholz, D. Nordlund et al., Orbital-Specific Mapping of the Ligand Exchange Dynamics of $\mathrm{Fe}(\mathrm{CO})_{5}$ in Solution, Nature (London) 520, 78 (2015).

[52] J. van den Brink and M. van Veenendaal, Correlation Functions Measured by Indirect Resonant Inelastic X-Ray Scattering, Europhys. Lett. 73, 121 (2006).

[53] F. Forte, L. J. P. Ament, and J. van den Brink, Magnetic Excitations in $\mathrm{La}_{2} \mathrm{CuO}_{4}$ Probed by Indirect Resonant Inelastic X-Ray Scattering, Phys. Rev. B 77, 134428 (2008).

[54] S. Kourtis, J. van den Brink, and M. Daghofer, Exact Diagonalization Results for Resonant Inelastic X-Ray Scattering Spectra of One-Dimensional Mott Insulators, Phys. Rev. B 85, 064423 (2012).

[55] J.-I. Igarashi and T. Nagao, Magnetic Excitations in L-Edge Resonant Inelastic X-Ray Scattering from One-Dimensional Cuprates, Phys. Rev. B 85, 064422 (2012).

[56] D. K. Morr, A. V. Chubukov, A. P. Kampf, and G. Blumberg, Raman Scattering in a Two-layer Antiferromagnet, Phys. Rev. B 54, 3468 (1996).

[57] N. Lin, E. Gull, and A. J. Millis, Two-Particle Response in Cluster Dynamical Mean-Field Theory: Formalism and Application to the Raman Response of High-Temperature Superconductors, Phys. Rev. Lett. 109, 106401 (2012).

[58] K. Tsutsui, H. Kondo, T. Tohyama, and S. Maekawa, Resonant Inelastic X-Ray Scattering Spectrum in High-Tc Cuprates, Phys. B Cond. Mat. 284-288, 457 (2000).

[59] T. Tohyama, K. Tsutsui, M. Mori, S. Sota, and S. Yunoki, Enhanced Charge Excitations in Electron-Doped Cuprates by Resonant Inelastic X-Ray Scattering, Phys. Rev. B 92, 014515 (2015).

[60] H. Y. Huang, C. J. Jia, Z. Y. Chen, K. Wohlfeld, B. Moritz, T. P. Devereaux, W. B. Wu, J. Okamoto, W. S. Lee, M. Hashimoto et al., Raman and Fluorescence Characteristics of Resonant Inelastic X-ray Scattering from Doped Superconducting Cuprates, Sci. Rep. 6, 19657 (2015).

[61] M. W. Haverkort, M. Zwierzycki, and O. K. Andersen, Multiplet Ligand-Field Theory Using Wannier Orbitals, Phys. Rev. B 85, 165113 (2012). 